\title{
SASI: A Generic Texture Descriptor for Image Retrieval
}

\author{
Abdurrahman Çarkacıoğlu ${ }^{l}$, Fatoş Yarman-Vural ${ }^{*}$ \\ Department of Computer Engineering, Middle East Technical University 06531 Ankara, Turkey
}

\begin{abstract}
In this paper, a generic texture descriptor, namely, Statistical Analysis of Structural Information (SASI) is introduced as a representation of texture. SASI is based on statistics of clique autocorrelation coefficients, calculated over structuring windows. SASI defines a set of clique windows to extract and measure various structural properties of texture by using a spatial multi-resolution method. Experimental results, performed on various image databases, indicate that SASI is more successful then the Gabor Filter descriptors in capturing small granularities and discontinuities such as sharp corners and abrupt changes. Due to the flexibility in designing the clique windows, SASI reaches higher average retrieval rates compared to Gabor Filter descriptors. However, the price of this performance is increased computational complexity.
\end{abstract}

Keywords: $\quad$ Texture Similarity, Image Retrieval, Clique, Autocorrelation, Descriptor

\section{INTRODUCTION}

In recent years, textural information has been widely used as a visual primitive in many image processing applications [1], [2], [3], [4]. The potential areas include industrial and biomedical surface inspection, ground classification and segmentation of satellite or aerial imagery, document analysis, scene analysis, texture synthesis for computer graphics and animation, biometric person authentication, content-based image retrieval and modelbased image coding [5], [6], [7].

Although the above application areas necessitate the utilization of texture analysis, only a limited number of successful interpretations of texture exist so far. The success of a texture descriptor heavily depends on the data type and the application area. A major problem in representing texture is that the textures in the real world are often quite

\footnotetext{
${ }^{1}$ His address: Sermaye Piyasasi Kurulu, Doc.Dr. Bahriye Ucok Cad.,No:13, 06500, Besevler/Ankara/TURKEY E-mail address: carkaci@,ceng.metu.edu.tr

* Her address: Department of Computer Engineering, Middle East Technical University, 06531,Ankara/TURKEY Tel: + 90-312-2104134; Fax: + 90-312-2101259

E-mail address: vural@,ceng.metu.edu.tr
} 
complex due to changes in orientation, scale or other visual appearance such as brightness and contrast [8]. Additionally, it is difficult to include extremely large number of attributes of texture under a single mathematical representation.

Texture, generally, refers to repetition of basic texture elements called texels [9]. Mathematically speaking, texture can be defined as stochastic, possibly periodic, two-dimensional image field. In practice, texture descriptors represent distinctive characteristics of a texture, which are specific to the problem domain. Unfortunately, none of the existing descriptors has been shown to give satisfactory results over a wide range of textures.

Textures can be represented by statistical, spectral and/or structural descriptors [9]. Well-known statistical descriptors are co-occurrence matrix, histogram features and random fields [10], [11], [9]. Gabor, Fourier and wavelet filters are the examples of spectral descriptors [12], [4]. Structural descriptors make use of texture primitives, where syntactic rules are employed for generating the texture [9]. Statistical descriptors exploit the local correlation of image pixels, whereas spectral descriptors capture global information about the energy on different scales. While statistical descriptors successfully analyze textures with weak edges or random nature, spectral and structural descriptors are best suited for periodic or almost periodic textures. In a given problem domain various types of textures may be mixed.

Currently, Gabor Filters are the most popular descriptors, used for texture similarity problems. Among many others [12], [13] the successful results are reported by Manjunath \& Ma [14], [15] where the image is first Gabor filtered, then, the second order statistics of the filter responses is used as a texture descriptor.

In [14] and [15] Manjunath and Ma compare Gabor Filter features with other texture features, namely, pyramidstructured wavelet transform (PWT) features, tree-structured wavelet transform (TWT) features and multiresolution simultaneous autoregressive model (MR-SAR) features, on the image retrieval problem by using Brodatz Album. They found that Gabor features slightly improves the overall performance by achieving an average retrieval rate close to $74 \%$ whereas MR-SAR features remains at $73 \%$.

Selection of the parameters for Gabor Filter descriptor depends on the characteristics of the textures in the image database. Since the Gabor functions are not orthogonal, there is a trade-off between redundancy and completeness 
in the design of the Gabor Filter Banks. Otherwise, the implementation of a complete Gabor expansion would entail a generally impractical number of filters. Also, in a digital world, it is not always possible to cope with all sizes of analog Gabor Filters, which may cause problems, especially, with the textures that consist of small texels or sharp corners. Another limitation of the Gabor descriptor is the restriction of the filtering area, which must fit in a rectangle, unless some pre-processing is done.

In this study, we explore a generic texture descriptor, which overcomes the above mentioned difficulties and works well on a wide range of textures. The SASI descriptor, proposed in this paper, is based on second order statistics of clique autocorrelation coefficients, which are the autocorrelation coefficients over a set of moving windows. The clique windows of various size and shape, which are defined by a neighborhood system, are used as a tool for describing the characteristics of textures in different granularity. The order of the neighborhood system controls the structure of the clique windows. Because of the flexibility in the definition of clique windows, SASI can cope with a broad class of textures, which may consist of discontinuities or small primitives.

SASI is tested on Brodatz Album [16], CUReT [17], PhoTex [18] and VisTex [19] image databases. The experiments are also performed on a combined database obtained by joining all of the images in these databases. It is observed that SASI improves the retrieval rates compared to Gabor Filters. However the price of this improvement is the increased computational complexity.

The paper is organized as follows. In Section 2, we introduce SASI descriptor behind a series of definition. Experimental results are given in Section 3. Section 4 concludes with discussions on the strengths and weaknesses of SASI compared to Gabor Filter descriptors.

\section{STATISTICAL ANALYSIS OF STRUCTURAL INFORMATION (SASI)}

\subsection{Definitions}

SASI is based on the concepts of clique [20] and autocorrelation coefficient. In the following, SASI descriptor is introduced along with the background definitions. 


\section{Definition 1: Neighboring set of a pixel}

For a regular lattice $\ell$, the neighboring set of a pixel $i j$ with coordinate $(i, j)$ is defined by the following recurrence relation:

$$
\begin{gathered}
\forall k l \in \ell, i j \neq k l, \\
\eta_{i j}^{d}=\eta_{i j}^{d-1} \bigcup \underset{\mathrm{kl} \notin \eta_{\mathrm{ij}}^{\mathrm{d}-1}}{\arg \min _{1}} D(i j, k l),
\end{gathered}
$$

and

$$
\eta_{i j}^{1}=\arg \min D(i j, k l)
$$

where $\quad \ell=\left\{i j \mid i, j \in \mathrm{N}, 1 \leq i \leq\right.$ Width $_{\ell}$ and $1 \leq j \leq$ Height $\left._{\ell}\right\}$,

$D(i j, k l)$ denotes the distance function between pixel $i j$ and $k l$,

$d$ is the order of neighborhood system and $d \in \mathrm{N}$.

The neighboring relationship has the following properties:

(1) a pixel is not neighboring to itself: $i j \notin \eta_{i j}^{d}$

(2) the neighboring relationship is commutative: $i j \in \eta_{k l}^{d} \Leftrightarrow k l \in \eta_{i j}^{d}$

Figure 1 shows the neighbors of pixel $i j$, where the labels from 1 to 5 indicate the order of the neighborhood system with respect to the Euclidean distance. In this figure, widely used first and second order neighborhood systems are indicated by label 1 and 2 , respectively.

Pixels near the edge of the lattice have fewer neighbors than the interior pixels. This fact is compensated by assuming that the lattice $\ell$ has a periodic or torus structure, which means that the left edge is connected to the right edge and the upper edge is connected to the lower edge [11], [21], [22], [23].

\section{Definition 2: Base Clique Type and Base Clique Test Predicate}

Given the neighborhood system $\eta^{d}$, base clique type set $\mathrm{P}$ is defined as,

$$
\mathrm{P}=\left\{\bar{p}=(i, j)-(k, l) \mid \forall k l \in \eta_{i j}^{d}\right\},
$$


Note that $\bar{p}$ is a position vector between two locations $(i, j)$ and $(k, l)$ of a lattice and called base clique type.

Figure 2 indicates the base clique types for the second order neighborhood system $\eta^{2}$, where $|P|=8$.

Base Clique Test Predicate $B_{\bar{p}}(i j, \mathrm{kl})$ is a Boolean function, which tests the neighboring and relative orientation of pixel $i j$ and $k l$ with respect to each other, given by:

$$
B_{\bar{p}}(i j, k l)=\left\{\begin{array}{ll}
\text { True } & \text { if }(k, l)-(i, j)=\bar{p} \text { and } k l \in \eta_{i j}^{d}, \\
\text { False } & \text { otherwise }
\end{array} \text { where } \bar{p} \in P .\right.
$$

Unlike neighboring relation, base clique test predicate is not commutative: $B_{\bar{p}}(i j, k l) \neq B_{\bar{p}}(k l, i j)$.

\section{Definition 3: Clique Chain}

Given pixel $i j$ as a seed, the clique chain $C_{L}^{\bar{p}}(i j)$ with length $L$ is a set defined by,

$$
\begin{aligned}
\forall \bar{p} \in P, \quad C_{L}^{\bar{p}}(i j)= & \{i j, k l, m n, \ldots, q r, s t, u v \mid \\
& \left.B_{\bar{p}}(i j, k l) \wedge B_{\bar{p}}(k l, m n) \wedge \ldots \wedge B_{\bar{p}}(q r, s t) \wedge B_{\bar{p}}(s t, u v)\right\},
\end{aligned}
$$

where total number of pixels in $C_{L}^{\bar{p}}(i j)$ is $L$,

$(i j, k l)$ and $(s t, u v)$ is the first and the last neighboring pair of pixels, with the base

clique type $\bar{p}$, respectively.

While in $\eta^{2}$, clique chains are lines of pixels with various directions, for higher order neighborhood systems, they become dash lines of pixels. Since $\eta^{2}=8$, only 8 direction clique chains can be obtained, as shown in Figure 3 . Note that each $C_{L}^{\bar{p}}(i j)$ is symmetric to $C_{L}^{-\bar{p}}(i j)$.

\section{Definition 3: Clique Window}

Clique Window $W_{S, L}^{\bar{p}, \bar{c}>}(a b)$ with seed $a b$ is an $S x L$ structuring element, which consists of S clique chains, defined as

$$
\begin{aligned}
W_{S, L}^{\bar{p}, \bar{c}>}(a b)=\left\{C_{L}^{\bar{p}}(a b) \bigcup C_{L}^{\bar{p}}(c d) \bigcup C_{L}^{\bar{p}}(e f) \bigcup \ldots \cup C_{L}^{\bar{p}}(w x) \bigcup C_{L}^{\bar{p}}(y z) \mid\right. \\
\left.B_{\bar{c}_{1}}(a b, c d) \wedge B_{\bar{c}_{2}}(c d, e f) \wedge \ldots \wedge B_{\bar{c}_{s-1}}(w x, y z) \quad\right\},
\end{aligned}
$$

where $a b, c d, e f, \ldots, w x, y z \in \ell,\langle\bar{c}\rangle$ is an ordered $\mathrm{S}-1$ tuple of base clique types such that $\left\langle\bar{c}>=<\bar{c}_{1}, \bar{c}_{2}, \ldots, \bar{c}_{S-1}>\right| \bar{c}_{i} \in P$, for $i=1 . . S-1$, and each element of $\left\langle\bar{c}>\right.$, denoted as $\bar{c}_{i}$, represents 
the base clique type of the $i^{t h}$ and $(i+1)^{t h}$ clique chain pair. It specifies how the clique chains, $C_{L}^{\bar{p}}$, are connected to each other. Parameters $\bar{p},\langle\bar{c}>, S$ and $L$ determine the structure of the clique window.

The clique window is called regular if $\bar{c}_{i}=\bar{c}_{j}$ for all $i, j=1 . . S-1$ and $\frac{\bar{c}_{i}}{\left|\bar{c}_{i}\right|} \neq \frac{\bar{p}}{|\bar{p}|}$ and $\frac{\bar{c}_{i}}{\left|\bar{c}_{i}\right|} \neq-\frac{\bar{p}}{|\bar{p}|}$, otherwise it is irregular.

Figure 4 illustrates some of the clique windows defined in $\eta^{2}$. In contrast to Figure 4 (a),(b),(c),(d) and (e), which are regular clique windows, Figure 4 (f),(g),(h) and (i) are the examples of irregular clique windows. For a regular clique window $W_{S, L}^{\bar{p}, \bar{c}>}(a b)$, the parameters $S, L$ and $, \bar{p},<\bar{c}>$ determine the size and orientation of the clique window, respectively. On the other hand, most of the time, it is hard to talk about the size and orientation of the irregular clique windows. This fact is depicted in Figure 4 (h). Thus, for the irregular clique windows, rather than the size and orientation, the structure becomes the main issue.

In this study, we mainly concentrate on the regular clique windows. For the sake of simplicity, $W_{S, L}^{\bar{p},<\bar{c}>}(a b)$ is abbreviated as $W_{S, L}^{\bar{p}, \bar{c}}(a b)$, since in regular clique windows, all $\bar{c}_{i} \mathrm{~s}$ are equal to each other. Also due to the symmetric relations a regular clique window $W_{S, L}^{\bar{p}, \bar{c}}(a b)$ has the same structure as $W_{S, L}^{-\bar{p}, \bar{c}}(a b), W_{S, L}^{\bar{p},-\bar{c}}(a b)$ and $W_{S, L}^{-\bar{p},-\bar{c}}(a b)$ as shown in Figure 5.

In $\eta^{2}, 12$ different clique windows (ignoring the symmetric ones) can be defined as shown in Figure 6. One can employ the higher order neighborhood systems, in order to incorporate the characteristics of the images in the database. Then, the clique windows can be defined based on the clique type set for a given neighborhood system. For example, 26, 86 and 124 regular clique windows can be defined in $\eta^{3}, \eta^{4}$ and $\eta^{5}$ respectively. Note that a clique window defined in $\eta^{d_{1}}$ can also be defined in $\eta^{d_{2}}$ if $d_{2} \geq d_{1}$.

In this study, only the regular clique windows are used for measuring the texture similarity. For practical reasons, the definitions below are given for regular clique windows. The generalization to irregular cases requires some extra works. 


\section{Definition 4: Clique Autocorrelation Coefficient}

Clique autocorrelation coefficient at lag vector $\bar{v}=\left(v_{x}, v_{y}\right)$ of a given seed pixel $a b$ for a regular clique window $W_{S, L}^{\bar{p}, \bar{c}}(a b)$ is given by

$$
r(\bar{v})^{W_{S, L}^{\bar{p} . \bar{c}}(a b)}=\frac{\sum_{\forall(i, j) \text { and }\left(i+v_{x} j+v_{y}\right) \in W_{S, L}^{\bar{p} \bar{c}}(a b)}\left(x_{i, j}-\bar{x}_{i, j}\right)\left(x_{i+v_{x} j+v_{y}}-\bar{x}_{i+v_{x} j+v_{y}}\right)}{\sqrt{\sum_{\forall(i, j) \in W_{S, \bar{c}}^{\bar{p} . \bar{c}}(a b)}\left(x_{i, j}-\bar{x}_{i, j}\right)^{2} \sum_{\forall\left(i+v_{x} j+v_{y}\right) \in W_{S, \bar{L}}^{\bar{p}, \bar{c}}(a b)}\left(x_{i+v_{x} j+v_{y}}-\bar{x}_{i+v_{x} j+v_{y}}\right)^{2}}}
$$

where $\quad x_{i, j}$ is the gray value of the image at position $(i, j)$ and

$$
\bar{x}_{i, j}=\frac{1}{N_{W_{S, L}^{\bar{p}, \bar{c}}(a b)}} \sum_{\forall(i, j) \in W_{S, \bar{L}}^{\bar{p} . \bar{L}}(a b)} x_{i, j}
$$

is the mean value of the gray levels and $N_{W_{S, L}^{\bar{c}, \bar{c}}(a b)}$ is the number of pixels in the clique window $W_{S, L}^{\bar{p}, \bar{c}}(a b)$.

Lag vector $\bar{v}$ is a vector between two locations of a clique window. Note that autocorrelation coefficients of a clique window depend only on the length and direction of the lag vector.

Clique autocorrelation coefficients can be considered as a short-term correlogram over the clique window defined by the clique chain. They enable us to capture stationary information at various scale and orientation within an image.

Since the autocorrelation coefficients at all lags bear redundant information as experimented later in Section 3.1.1, there is no need to use all of them for the representation of a texture in a multidimensional space. Therefore, it is reasonable to take the lag vector $\bar{v}$ of a clique window $W_{S, L}^{\bar{p}, \bar{c}}(a b)$ as,

$$
\bar{v}=n \times \bar{p}=\left(n p_{x}, n p_{y}\right)
$$

where $n$ is a lag multiplier and $n \in \mathrm{N}, 1 \leq n \leq \mathrm{L}-1$. In other words, the lag vector $\bar{v}$ is taken as the same as the base clique type of the clique chains, which make the clique window.

\section{Definition 5: Second Order Statistics of Autocorrelation Coefficients}

Mean value and standard deviation of clique autocorrelation coefficients with lag vector $\bar{v}$ of all clique windows $W_{S, L}^{\bar{p}, \bar{c}}(a b)$ is defined as 


$$
\mu_{\mathrm{S}, \mathrm{L}}^{\overline{\mathrm{p}} \overline{\mathrm{c}}}(\bar{v})=\frac{1}{N_{\ell}} \sum_{\forall(a, b) \in \ell} r(\bar{v})^{W_{S, L}^{\overline{\bar{p}} \bar{c}}(a b)}
$$

and

$$
\sigma_{\mathrm{S}, \mathrm{L}}^{\overline{\mathrm{p}}, \overline{\mathrm{c}}}(\bar{v})=\sqrt{\frac{1}{N_{\ell}} \sum_{\forall(a, b) \in \ell}\left(r(\bar{v})^{W_{S, \bar{L}}^{\overline{\bar{p}}, \bar{c}}(a b)}-\mu_{\mathrm{S}, \mathrm{L}}^{\overline{\mathrm{p}}, \overline{\mathrm{c}}}(\bar{v})\right)^{2}}
$$

respectively, where $\bar{v}$ is the lag vector, $\bar{p}$ and $\bar{c}$ are the base clique types, $S$ is the number of clique chain, $L$ is the clique chain length and $N_{\ell}$ is the number of pixels in the lattice.

\section{Definition 6: SASI Descriptor}

For a given texture T, SASI descriptor is defined as an Nx1 vector with the entries $\mu_{\mathrm{S}, \mathrm{L}}^{\overline{\mathrm{p}}, \overline{\mathrm{c}}}(\mathrm{n}), \sigma_{\mathrm{S}, \mathrm{L}}^{\overline{\mathrm{p}}, \overline{\mathrm{c}}}(\mathrm{n})$ as

$$
\mathrm{D}_{\mathrm{T}}=\left\{\mu_{\mathrm{S}_{1}, \mathrm{~L}_{1}}^{\overline{\mathrm{p}}_{1}, \overline{\mathrm{v}}_{\mathrm{1}}}\left(\bar{v}_{1}\right), \ldots, \mu_{\mathrm{S}_{Q}, \mathrm{~L}_{Q}}^{\overline{\mathrm{p}}_{Q}, \overline{\mathrm{c}}_{\mathrm{Q}}}\left(\bar{v}_{Q}\right), \sigma_{\mathrm{S}_{1}, \mathrm{~L}_{1}}^{\overline{\mathrm{p}}_{1}, \overline{\mathrm{v}}_{1}}\left(\bar{v}_{1}\right), \ldots, \sigma_{\mathrm{S}_{Q}, \mathrm{~L}_{Q}}^{\overline{\mathrm{p}}_{Q}, \overline{\mathrm{c}}_{\mathrm{Q}}}\left(\bar{v}_{Q}\right)\right\}
$$

where $2 \times Q(Q$ mean values $+Q$ standard deviations $)$ is the size of the feature vector.

For each selected clique window $W_{S_{i}, L_{i}}^{\bar{p}_{i} \bar{c}_{i}}(a b)$, where $i=1 \ldots$ total_number_of_clique_windows_selected, total number of mean value and standard deviations calculable are $2 \times\left(L_{i}-1\right)$, since lag vector $\bar{v}$ is defined as $\bar{v}=n \times \bar{p}_{i}$ where $n \in \mathrm{N}, 1 \leq n \leq \mathrm{L}_{\mathrm{i}}-1$. Therefore, the maximum value of $Q$ is $\sum_{i=1}^{\text {Total \# of Clique Window }}\left(L_{i}-1\right)$.

\section{Definition 7: Normalized SASI Descriptor}

Given, $D_{T}=\left\lfloor f_{1}, f_{2}, \ldots, f_{2^{*} Q}\right\rfloor$, then normalized SASI descriptor, $D_{T}^{\prime}=\left\lfloor f_{1}^{\prime}, f_{2}^{\prime}, \ldots, f_{2^{*} Q}^{\prime}\right\rfloor$, is defined by normalizing the entries of $\mathrm{D}_{\mathrm{T}}$ as follows:

$$
f_{i}{ }^{\prime}=\frac{f_{i}-\mu_{f_{i}}}{\sigma_{f_{i}}} \quad \mathrm{i}=1 . .2 * Q
$$

where $\mu_{f_{i}}$ is the mean value and $\sigma_{f_{i}}$ is the standard deviation of the features over the entire database. 
$\mathrm{D}_{\mathrm{T}}^{\prime}$ measures the structural information by using the second order statistics of local autocorrelation coefficients for texture $\mathrm{T}$. The size of the descriptor $\mathrm{D}_{\mathrm{T}}^{\prime}$ depends on the image database. We use the distance metric defined below in order to measure the mathematical similarity between the textures.

\section{Definition 8: SASI Distance}

In this study, the mathematical similarity between the textures $\mathbf{T}_{\mathbf{1}}$ and $\mathbf{T}_{\mathbf{2}}$ is measured by the following metric:

$$
S\left(T_{1}, T_{2}\right)=\frac{D_{T_{1}}^{\prime} \bullet D_{T_{2}}^{\prime}}{D_{T_{1}}^{\prime} \bullet D_{T_{1}}^{\prime}+D_{T_{2}}^{\prime} \bullet D_{T_{2}}^{\prime}-D_{T_{1}}^{\prime} \bullet D_{T_{2}}^{\prime}}
$$

where $\bullet$ stands for dot product.

\subsection{Algorithm of SASI}

Begin

Select neighborhood system $\eta^{d}$

Select the clique windows $W_{S, L}^{\bar{p}, \bar{c}}$ as a subset of all the clique windows

Select the lag vectors used for each clique window

$D_{T}=\phi$

For each clique window $W_{S, L}^{\bar{p}, \bar{c}}$

For each lag vector $\bar{v}$

For each pixel $(a b)$

Define clique window $W_{S, L}^{\bar{p}, \bar{c}}(a b)$

Calculate $r(\bar{v})^{W_{S, L}^{\bar{p}, \bar{c}}(a b)}$ using Equation (1)

Next pixel $(a b)$

Calculate mean value $\mu_{\mathrm{S}, \mathrm{L}}^{\overline{\mathrm{p}}, \overline{\mathrm{c}}}(\bar{v})$ and standard deviation $\sigma_{\mathrm{S}, \mathrm{L}}^{\overline{\mathrm{p}}, \overline{\mathrm{c}}}(\bar{v})$

$$
D_{T}=D_{T} \cup\left\{\mu_{\mathrm{S}, \mathrm{L}}^{\overline{\mathrm{p}}, \overline{\mathrm{c}}}(\bar{v}), \sigma_{\mathrm{S}, \mathrm{L}}^{\overline{\mathrm{p}}, \overline{\mathrm{c}}}(\bar{v})\right\}
$$

Next $\bar{v}$

$\operatorname{Next} W_{S, L}^{\bar{p}, \bar{c}}$

End.

Construct normalized $D_{T}^{\prime}$ vector using Equation (5)

The most crucial part of the algorithm is the selection of the clique window sizes, $S$ and $L$. A preliminary analysis on database, as discussed in the next section, may help us to determine them. Window sizes depend on the size of the texture primitives and resolution of the images in the database. Basically, clique windows should be small enough to capture small primitives and big enough to capture large patterns or primitives in the images of the database. One can employ all possible size clique windows and related autocorrelation coefficients, but this time, 
the computational power is wasted. Additionally, increasing the dimension of the feature vector may not improve the representation capability of the descriptor. This is a well-known phenomenon, called "curse of dimensionality", in pattern recognition.

\section{EXPERIMENTS}

Two sets of experiments are done to show the power of SASI. First, SASI descriptor is analyzed in detail and compared to Gabor Filter descriptor. Latter, SASI and Gabor Filter descriptor are tested on the image retrieval problem by using four different image databases, namely Brodatz Album, CUReT [17], PhoTex [18] and VisTex [19]. The experiments are also performed on a database generated by joining all the images of these four databases.

Brodatz Album contains 112 pictures with size 512x512 and 256 gray values after digitizing, showing a variety of textures, collected for artistic purposes [16]. It is a de facto standard set of images for texture retrieval problem. Due to its popularity and comparable studies exist in the literature [24], [14], [15]; a more comparative analysis is provided on the Brodatz Album in the following sections.

Columbia-Utrecht Reflectance and Texture Database (CUReT) are formed by the researchers at Colombia University and Utrecht University [17]. It contains 61 different pictures with various size and color. Thumbnails of the images can be seen at http://www.cs.columbia.edu/CAVE/curet/html/sample.htm. Before applying SASI and Gabor descriptors, each image is rescaled to $512 \times 512$ and converted to gray scale.

Jerry Wu from Heriot-Watt University at Edinburgh creates Photometric Texture Database (PhoTex) [18]. 30 different pictures with size $512 \times 512$ and 256 gray values exist in PhoTex. Images can be seen at http://www.cee.hw.ac.uk/texturelab/database/jwdb/thumbnails.htm as thumbnails.

Vision Texture Database (VisTex) is formed by the Vision and Modeling group at the MIT Media Lab [19]. It contains 167 colored reference textured images with size $512 \times 512$. Images are grouped according to their contents. Further details can be found at http://www-white.media.mit.edu/vismod/imagery/VisionTexture/vistex.html. 


\subsection{SASI in Detail}

In this section, first, traditional correlogram method is examined to show the redundancy in autocorrelation coefficients in the analysis of texture. Next, the clique windows are employed in order to show the effect of the window sizes in constructing the SASI descriptor. Then, SASI descriptor is compared to Gabor Filter descriptor.

\subsubsection{Traditional Correlogram Analysis}

Traditional correlogram is a special case of SASI, where the size and the shape of the clique windows are chosen as the size and the shape of the image itself and clique autocorrelation coefficient is calculated for all lag vectors. The resulting series is called the autocorrelation series or correlogram.

Figure 7 shows the correlogram of texture D001 from Brodatz Album. The correlogram in Figure 7 shows that at each 30 pixel in $\mathrm{X}$ and $\mathrm{Y}$ direction texture primitives are repeated.

It is well known that correlogram bears redundant information [9], [25], [26]. This redundancy is partially observed by using Principal Component Analysis. When the rows of the correlogram are chosen as variables, it can be seen that few new variables or principal components are sufficient to capture the information provided by the existing variables. This fact is exemplified in the sample Brodatz texture, D001 in Table 1. Note that only 5 principal components are sufficient to capture almost all the information provided by 129 variables.

A similar analysis indicates that there is no need to calculate all lags of the clique autocorrelation coefficient for determining the SASI descriptor. Thus, as mentioned in Definition 4, lag vector $\bar{v}$ of a clique autocorrelation $r(\bar{v})^{W_{S, L}^{\bar{p} . \bar{c}}(a b)}$ is taken as, $\bar{v}=n \times \bar{p}$, where $n$ is a lag multiplier.

In the next section the effect of window size parameters $S, L$ on texture representation will be explored in detail.

\subsubsection{Selection of Clique Window Size in SASI Descriptor}

In order to analyze clique window size, four regular clique windows, namely $W_{S=L}^{(1,0),(0,1)}, W_{S=L}^{(0,1),(1,0)}, W_{S=L}^{(1,1),(1,0)}$ and $W_{S=L}^{(-1,1),(1,0)}$ are defined, as shown in Figure 8, which are horizontal, vertical, right and left diagonal clique 
windows, respectively. For each clique window, possible lag vectors $\bar{v}$ for the clique autocorrelation coefficients are also shown.

For the notational simplicity, horizontal, vertical, right diagonal, left diagonal clique windows will be represented as $W_{S}^{H}, W_{S}^{V}, W_{S}^{R D}$ and $W_{S}^{L D}$. Working with these clique windows may help us to analyze the effects of the clique window sizes and the clique autocorrelation lags on SASI.

Different properties or components of the texture are captured by the clique autocorrelation coefficients at different lag vectors applied on the clique windows. Figure 9 illustrates the relation between the clique window size versus mean values and standard deviations of autocorrelation coefficients of texture D001 in the Brodatz Album. Note that the mean values and standard deviations of the autocorrelation coefficients remain almost the same for larger values of clique window than the size $25 \times 25$. Therefore, for this particular example, it is shown that using clique window size larger than $25 \times 25$ does not bring any critical information. Similar analysis is done for the texture D052 shown in Figure 10. In this case, the largest window size might be 15x15.

In Figure 9 and 10, the clique autocorrelation coefficients are calculated for 4 orientations of clique windows with the lag multiplier $n=1$. Whereas in Figure 11 and 12, directions of lag vectors are fixed, but varying lag multiplier $n$ is employed for textures D001 and D052. Due to the dominant horizontal and vertical effects in texture D001 and dominant diagonal effects in texture D052, related clique windows are selected to examine the dominant features of both textures. Figure 11 and 12 indicates that using clique window size larger than $33 \times 33$ for texture D001 and larger than 19x19 for texture D052 brings very small information for clique autocorrelation coefficient with lag multiplier $n=2$.

Note that, the mean value of the clique autocorrelation coefficients approaches to the autocorrelation coefficient of the entire texture, as the size of the clique window gets larger.

The window sizes and the lag vectors of the autocorrelation coefficients for each clique window are the critical parameters of SASI. Therefore, a preliminary analysis on the images of the database is required to select these parameters before the calculation of SASI descriptor. 


\subsubsection{Gabor versus SASI Descriptor}

Gabor Filter descriptor, reported by Manjunath and Ma [14], [15] use a dictionary, which contains four scales and six orientations. Each filter captures the relevant texture primitives of the image. Second order statistics of the Gabor Filter $(4$ scales $\times 6$ orientation $=24$ filter $)$ responses of a given texture is used as a texture descriptor. Thus, an image is represented by a vector $\vec{f}$ of size 48 [15].

$$
\vec{f}=\left\{\mu_{00}, \sigma_{00}, \ldots, \mu_{(S-1)(K-1)}, \sigma_{(S-1)(K-1)}\right\},
$$

where the subscript $S$ represents the scale $(S=0, . ., 3)$ and $K$ represents the orientation $(K=0, . ., 5)$. The distance between two images is defined as:

$$
d(i, j)=\sum_{S} \sum_{K}\left|\frac{\mu_{S K}^{i}-\mu_{S K}^{j}}{\alpha\left(\mu_{S K}\right)}\right|+\left|\frac{\sigma_{S K}^{i}-\sigma_{S K}^{j}}{\alpha\left(\sigma_{S K}\right)}\right|,
$$

where $\alpha\left(\mu_{S K}\right)$ and $\alpha\left(\sigma_{S K}\right)$ are the standard deviations of respective features over the entire database.

The algorithmic complexity of Gabor Filter descriptor is $O\left(N \times \log _{2} N\right)$ when filtering is implemented in frequency domain, whereas the complexity of SASI is $O(S \times L \times N)$ where $\mathrm{S} \times \mathrm{L}$ is a clique window size and N is the image size and $\mathrm{N}=$ Width $\times$ Height of the image. If the size of the clique window is small compared to the image size ( $\left.S \times L<\log _{2} N\right)$ than SASI descriptor requires less computational power than that of Gabor Filter. However in our experiments, we use clique windows of size $3 \times 3,5 \times 5$ and $7 \times 7$ for $128 \times 128$ images. Therefore, for the databases used in this study, Gabor Filter is more efficient in terms of the computational complexity.

As in Gabor Filter, SASI captures the components of texture with different coarseness. As a result, coarse-to- fine components of the textures are represented in large-to-small size clique windows. This fact is depicted in Figures 13, 14 and 15 where various sizes of clique windows decompose the image into various granularities.

In order to depict the characteristics of both SASI and Gabor Filter descriptors, three textures are selected from the Brodatz Album, namely D001, D035 and D052. D001 contains sharp edges with large texels and high contrast. On the other hand, D035 and D052 have coarse to fine textures with relatively low contrast. Considering the structure of the textures, D001 is analyzed by horizontally oriented clique windows and Gabor Filters, whereas D035 is 
analyzed by diagonal clique windows and Gabor Filters, since these effects are dominant in the selected textures. Also, the vertical effects are analyzed in D052, which consists of vertical, diagonal and horizontal texels.

Table 2 indicates the parameters of Gabor and SASI descriptors used in the experiment. The outputs of the clique autocorrelation coefficients and the Gabor Filter responses shown in Figure 13, 14 and 15 are scaled to 0 to 255, where 255 (white) and 0 (black) correspond to the high and low responses, respectively.

The results of the filter responses and the clique autocorrelation coefficients depicted in Figure 13, 14 and 15 are not directly comparable. However, by analyzing these figures, one can get an idea about how these two descriptors work. Although there is no one to one mathematical match, for each image, small to big clique window versus narrow to wide Gabor filter is employed.

A comparison of SASI and Gabor Filter outputs in Figure 13 indicates that while SASI captures the sharp edges, Gabor has a tendency to smooth them. The Gaussian structure of the Gabor Filter naturally, bends the straight lines while SASI captures them without any deformation.

It can be seen from Figure 14 and 15 that, Gabor Filter fails to capture small texels because of the error in discrete approximation of Gabor function for small windows. The output of fine parameters of Gabor is almost white noise (no pattern).

\subsection{Image Retrieval}

Textural information can be used in two main application domains: 'between-image search' and 'within-image search'. The first domain deals with searching an image database and finding the most similar image to a given query image. The latter deals with texture segmentation problem, searching a region within an image and finding the most similar region to a given object or a region. Although the proposed descriptor can be used in both domains, in this study, we are mainly concentrated on between-image search problem since in this domain, the performance of a descriptor can be easily evaluated in terms of the average retrieval rates [24], [27], [14], [2], [15]. On the other hand, the concept of similarity is quite subjective. 
There are two popular methods for testing the performance of a texture descriptor:

i. Each image in the database is divided into sub-images,

ii. The images in the database are grouped by the user.

The first method enables us to identify each subimage without human subjective support, unless images are similar to each other, whereas the latter method requires grouping criteria that may differ from user to user. Although human support adds subjectivity to the performance measuring process, without this support human visual system consistency of a descriptor cannot be fully measured. This is a dilemma of the performance measuring process.

\subsubsection{Image Retrieval without Human Subjectivity}

In our experiments, all of the images in Brodatz Album, CUReT, PhoTex and VisTex databases are partitioned into 16 nonoverlapping regions, as shown in Figure 16. Hence, for Brodatz Album 112×16=1792 subimages, for CUReT, $61 \times 16=976$ subimages, for PhoTex $30 \times 16=480$ subimages and for VisTex $67 \times 16=2672$ subimages are obtained, as shown in Table 3. The performance of the proposed descriptor for each image database is measured in terms of the average retrieval rate, which is defined as the average percentage number of patterns belonging to the same image as the query pattern in top 15 matches (self matches are excluded) [14], [15], [24]. In another words, for each subimage, its most similar 15 subimages are searched within the entire database consists of subimages. In the ideal case, retrieved 15 closest and the query subimage should come from the same original image. This type of performance appraisal is widely used in between-image search applications.

Throughout the image retrieval experiments $3 \times 3,5 \times 5$ and $7 \times 7$ clique windows are employed. Table 4 (a) shows the autocorrelation coefficients and the related window sizes, which are selected in the preliminary analysis of Brodatz, CUReT, PhoTex and VisTex image databases, as explained in the previous section. As it can be seen from the Table 4 (a) for a given clique window type, 10 autocorrelation coefficient are calculated and the feature vector of size 20 (10 mean value +10 standard deviation $)$ is formed.

In order to make a systematic analysis on the performance of SASI descriptor all clique windows defined in $\eta^{1}$, $\eta^{2}, \eta^{3}, \eta^{4}$ and $\eta^{5}$ are employed. Table 4 (b) shows the number of clique windows and corresponding feature vector size for each neighborhood system. 
After calculating the SASI descriptor, the ranking process is accomplished by using Equation (6).

The average retrieval rates of Gabor and SASI descriptor are computed for images in Brodatz, CUReT, PhoTex and VisTex databases, respectively and the results are indicated in Table 3. Note that SASI descriptor achieves average retrieval rate between $47-92 \%$ whereas Gabor Filter remains in the range of $46-80 \%$. We, also, formed a large image database by combining all the subimages of the Brodatz, CUReT, PhoTex and VisTex. In this experiment, for each subimage its most similar 15 subimages are searched within 5,920 subimages. The average retrieval rate for SASI descriptor (in $\eta^{3}$ ) is $67.20 \%$ whereas that of Gabor is $60.56 \%$.

In SASI increasing the order of the neighborhood system larger than 3, decreases the average retrieval rate, due to curse of dimensionality. Thus, the below experiments are done by using SASI with clique windows defined in $\eta^{3}$.

Although we did not perform a systematic set of experiments to check the consistency of SASI to the human visual system, during the experiments, we observed that SASI retrieves images which are quite consistent to our intuition. In order to show these informal results, two examples, where 4 query texture and their 35 closest textures in the Brodatz Album, are given in Appendix-Figure 1.a and 1.b.

The retrieval rate for each image in the Brodatz album for Gabor and SASI descriptor is shown in Table 5. Figure 17 indicates the percentage of retrieving the correct subimages as a function of number of retrieved subimages. In Figure 17, horizontal axis represents the number of retrieved subimages and vertical axis represents the percentage of the correct retrieved subimages. The performance increases to $93 \%$ if the top 100 retrievals are considered instead of 15 retrieval considerations.

\subsubsection{Image Retrieval with Human Help: Clustering}

Brodatz, CUReT, PhoTex and VisTex image databases were never intended to give a fully representative sample set of a broad class of textures for testing the full performance of texture descriptors. As described earlier, during the evaluation of the performance of a descriptor, the images of the databases are partitioned into $n$ subimages. Then, for each subimage, its most similar $n-1$ subimages are searched within the subimages in the database. In this case, images can be considered as distinct classes, whereas the subimages correspond to the entries of each class. 
It is expected that the query and the retrieved most similar, i.e. closest, $n-1$ subimages are regions of the same image. This expectation is only valid for an image database, where images of the database are visually different from each other whereas the subimages are visually similar. However, the databases used in the experiments are far from satisfying this expectation.

There are two major problems for measuring the performance of a descriptor. Firstly, some images in the database are quite similar to each other. Secondly, splitting an image into subimages may sometimes yield visually dissimilar textures. These problems prevent us to measure the consistency of a descriptor with the human visual system.

In order to avoid the above problems, the subimages may be clustered by the human support. However, in this case the measured performance of a descriptor is human specific. Also, when the number of images clustered by the human is increased, subjectivity is also increased from one person to the other.

Since it is hard to manually group the subimages, 112 textured images of Brodatz Album are visually grouped into 32 different clusters, each of which contains 1-8 similar texture [15], [24], [27]. In this study, we use the clustering schema defined in [24] as shown in Table 6. After the grouping, each image is partitioned into 16 subimages. Note that, this clustering process can eliminate the problems of Brodatz Album mentioned above, to a certain extent.

This time, the query and retrieved most similar subimages are tested for belonging to the same cluster. Since clusters contain different number of images rather than average retrieval rates, weighted average retrieval rates, where the weights are the number of images in each cluster, are considered. Figure 18 illustrates an evaluation based on 32 clusters. Weighted average retrieval rate of SASI descriptor is higher than that of Gabor Filter descriptor. As it can be seen from Figure 18, when clustering is employed, the most similar 8 subimages of a given query subimage are in the same cluster at the rate of $90 \%$.

As stated in Section 3, images in the VisTex database were grouped according to their contents by the researchers at the MIT Media Lab. In table 7, 19 groups of images in the VisTex are shown. Same analysis defined in the previous paragraph is applied on visually grouped images of VisTex. Figure 19 shows an evaluation based on 19 clusters where the most similar 8 subimages of a given query subimage are in the same cluster at the rate of $85 \%$. 


\section{CONCLUSUIONS}

In this paper, a new texture descriptor, namely SASI, is introduced and compared to Gabor Filters. SASI descriptor consists of second order statistics of autocorrelation coefficient at different lags over a set of clique windows. The concept of clique chain is employed for constructing these structural windows. Clique windows are defined by using a set of neighborhood systems. Changing the order of the neighborhood system, various regular or irregular clique windows are generated. The size of the clique windows and the lag vectors for the autocorrelation coefficients are the parameters of SASI. Selection of these parameters requires domain dependent analysis. The traditional correlogram is a special case of SASI, where only one clique window is used with the size of the image itself and clique autocorrelation coefficient is calculated for all lag vectors. Therefore, SASI can be considered as a generalized correlogram.

SASI descriptors have various superiorities compared to Gabor filters. First of all, the Gaussian structure of the Gabor filters, has the tendency to bend the straight lines and smoothes the sharp edges. On the other hand, the flexibility in designing a large class of clique windows enables one to capture a great variety of textures without any distortion. Secondly, while SASI descriptors can successfully extract small texels, the Gabor function fails to detect them, due to the relatively large error of the discrete filter approximation. As a result, SASI descriptor captures the structural property of the texture better than the Gabor Filters. This fact is verified during the performance tests based on average retrieval rates applied on subimages and visually clustered images of Brodatz Album, CUReT, PhoTex and VisTex databases. Finally, during the experiments it is observed that SASI descriptor is more consistent to the Human Visual System compared to the Gabor filters, in retrieving the similar images. This is quite reasonable considering the fact that SASI does not restrict the textures to obey the laws of Gaussian nature. On the other hand, the main disadvantage of SASI descriptor is its high computational complexity, especially for large size clique windows required for capturing large texels. 


\section{REFERENCES}

[1] A.K. Jain, S.K. Bhattacharjee, and Y. Chen. "On texture in document images", Proceedings of Computer Vision and Pattern Recognition, pages 677-680,1992

[2] P. Wu, B. S. Manjunath, S. Newsam and H.D. Shin, " A texture descriptor for browsing and similarity retrieval," Journal of Signal Processing: Image Communication, Volume 16, Issue 1-2, page 33-43, September 2000

[3] A. Pentland, R.W. Picard and S.Sclaroff, "Photobook: tools for content based manipulation of image databases" Proc. of SPIE, Storage and Retrieval for Image and Video Databases-II No.2185, 34-47, Feb. 1994.

[4] Y. Rui and T. Huang and S. Chang, "Image Retrieval: Current Techniques, Promising Directions And Open Issues", Journal of Visual Communication and Image Representation, Vol. 10, no. 4, pp. 39-62, April 1999

[5] A. Asona and M. Miyagawa and M. Fujio, "Texture Modelling by Optimal Gray Scale Structuring Elements Using Morphological Pattern Spectrum", ICPR, Vol III: 479-482, 2000

[6] A.K. Jain and Aditya Vailaya,"Image retrieval using color and shape",Pattern Recognition, 29(8):1233--1244, August 1996.

[7] M Pietikäinen,T. Ojala \& Z. Xu ,"Rotation-invariant texture classification using feature distributions", Pattern Recognition Vol. 33:43-52,2000

[8] D. Harwood, T. Ojala, M. Pietikäinen, S. Kelman \& L. Davis, "Texture classification by center-symmetric autocorrelation, using Kullback discrimination of distributions", Pattern Recognition Letters 16:1-10,1995

[9] A.K. Jain, "Fundementals of Digital Image Processing", Prentice Hall, 1989

[10] T.R. Reed and J.M.H.du Buf, “A Review of Recent Texture Segmentation and Feature Extraction Techniques”, CVGIP: Image Understanding, vol57, and pp.359-372, May 1993.

[11] G. Cross and A.K.Jain, "Markov random field texture models”, IEEE Trans. On PAMI., vol. 1-5, pp. 25 - $39,1983$.

[12] A.K. Jain and F.Farroknia,“Unsupervised texture segmentation using Gabor filters”,Pattern Recognition, 24(12), 11671186, 1991.

[13] A. Jain, N. Ratha and S. Lakshmanan, "Object detection using Gabor filters", Pattern Recognition, Vol. 30, 295-309,1997

[14] W.Y.Ma and B.S.Manjunath,“Texture features and learning similarity,” Proc. IEEE International Conference on Computer Vision and Pattern Recognition, San Francisco, CA, pp. 425-430, June 1996

[15] B.S. Manjunath and W.Y. Ma, “Texture features for browsing and retrieval of image data,” IEEE Trans. On PAMI, Vol. 18, No. 8, pp 837-842, Aug. 1996.

[16] P.Brodatz: "Textures, A photographic album for artists and designers", Dover Publications, New York, 1966.

[17] K. J. Dana, B. van Ginneken, S. K. Nayar, J. J. Koenderink,"Reflectance and texture of real world surfaces", ACM Transactions on Graphics,pp 1-34, January, 1999

[18] J. Wu, "Investigation the Use of Photometric Stereo for Surface Rotation-Invariant Texture Classification", technical report RM/99/01, Department of Computing \& Electrical Engineering, Heriot-Watt University, Edinburgh, August 1999.

[19] R. Picard, C.Graczyk, S.Mann, J.Wachman, L.Picard and L.Campbell. "Vistex". via ftp:whitechapel.media.mit.edu, 1995. Copyright 1995 Massachusetts Institute of Technology.

[20] S. Z. Li. "Markov Random Field Modeling in Computer Vision", Springer-Verlag, 1995

[21] A. Carkacioglu and F.T. Yarman-Vural, "Set of Texture Similarity Measures", Electronic Imaging 97, Machine Vision Applications in Industrial Inspection, SPIE Proceedings Vol. 3029, pp. 118-127, San Jose, 1997.

[22] A. Carkacioglu and F.T. Yarman-Vural, "A Set of Similarity Measures for Binary and Gray Level Markov Random Fields Textures", IAPR - ICIAP Vol. 2, 127-133, Italy 1997.

[23] A.Carkacioglu and Fatos Yarman-Vural "SASI: A New Texture Descriptor for Image Retrieval", IEEE,International Conference of Image Processing, , pp. 137-140, Thesselenoki, Greece, 2001

[24] G. Guo, S.Z. Li, and K.L.Chan, "Learning Similarity for Texture Image Retrieval", Proceedings of the European Conference on Computer Vision Dublin, pp. 178-190, Ireland, June 2000.

[25] C. Chatfield, "The Analysis Of Time Series", Chapman and Hall, 1989

[26] Trevor C. Bailey \& Anthony C. Gatrell, "Interactive Spatial Data Analysis.” Longman, Essex, 1995

[27] G.Guo, H.Zhang and S.Z.Li, "Distance from boundary as a metric for texture image retrieval", Proc. of ICASSP, Salt Lake City, May 2001. 


\begin{tabular}{|l|l|l|l|l|}
5 & 4 & 3 & 4 & 5 \\
\hline 4 & 2 & 1 & 2 & 4 \\
\hline 3 & 1 & $\mathrm{ij}$ & 1 & 3 \\
\hline 4 & 2 & 1 & 2 & 4 \\
\hline 5 & 4 & 3 & 4 & 5 \\
\hline
\end{tabular}

Figure 1. Neighbors of pixel $i j$. The labels $\mathrm{d}=1, \ldots, 5$ indicate the order of neighborhood system with respect to Euclidean distance.

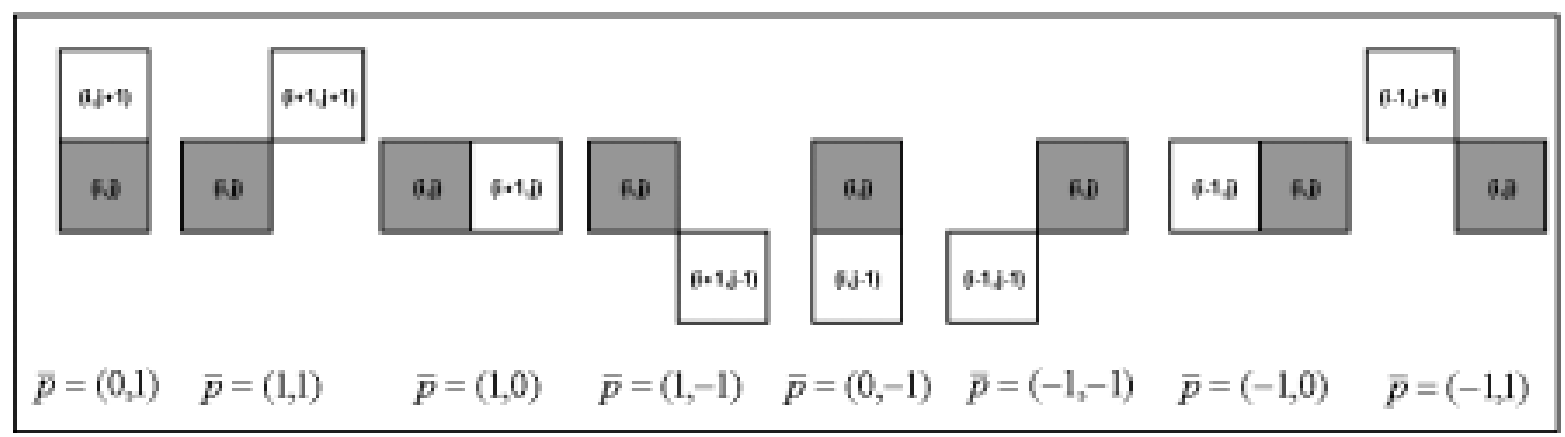

Figure 2. Base clique types $\bar{p}$, in $\eta^{2}$ neighborhood. Shaded pixel is taken as a seed pixel. 


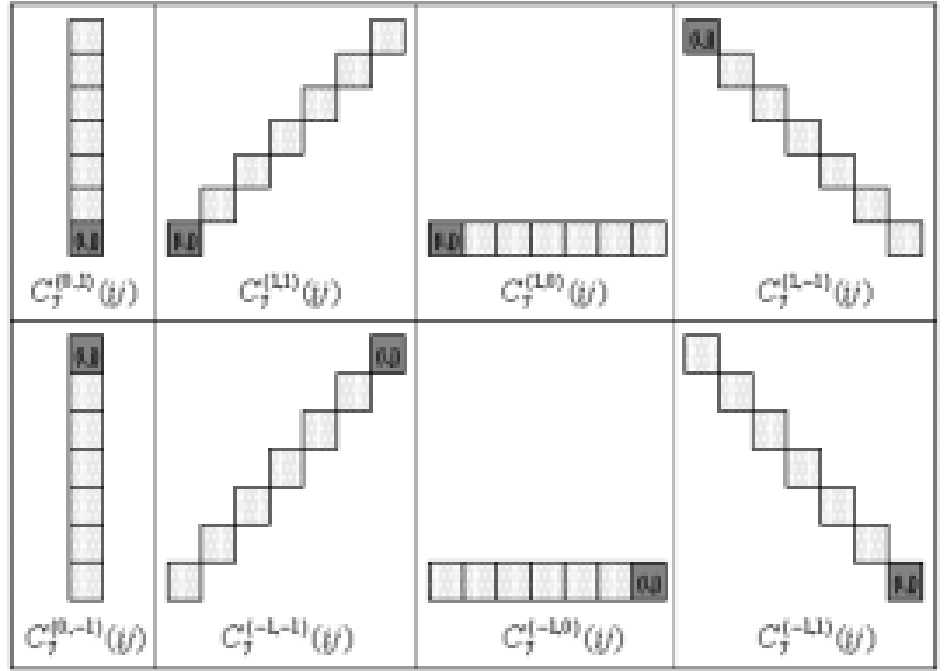

Figure 3. 8 orientations of clique chain with length 7 .

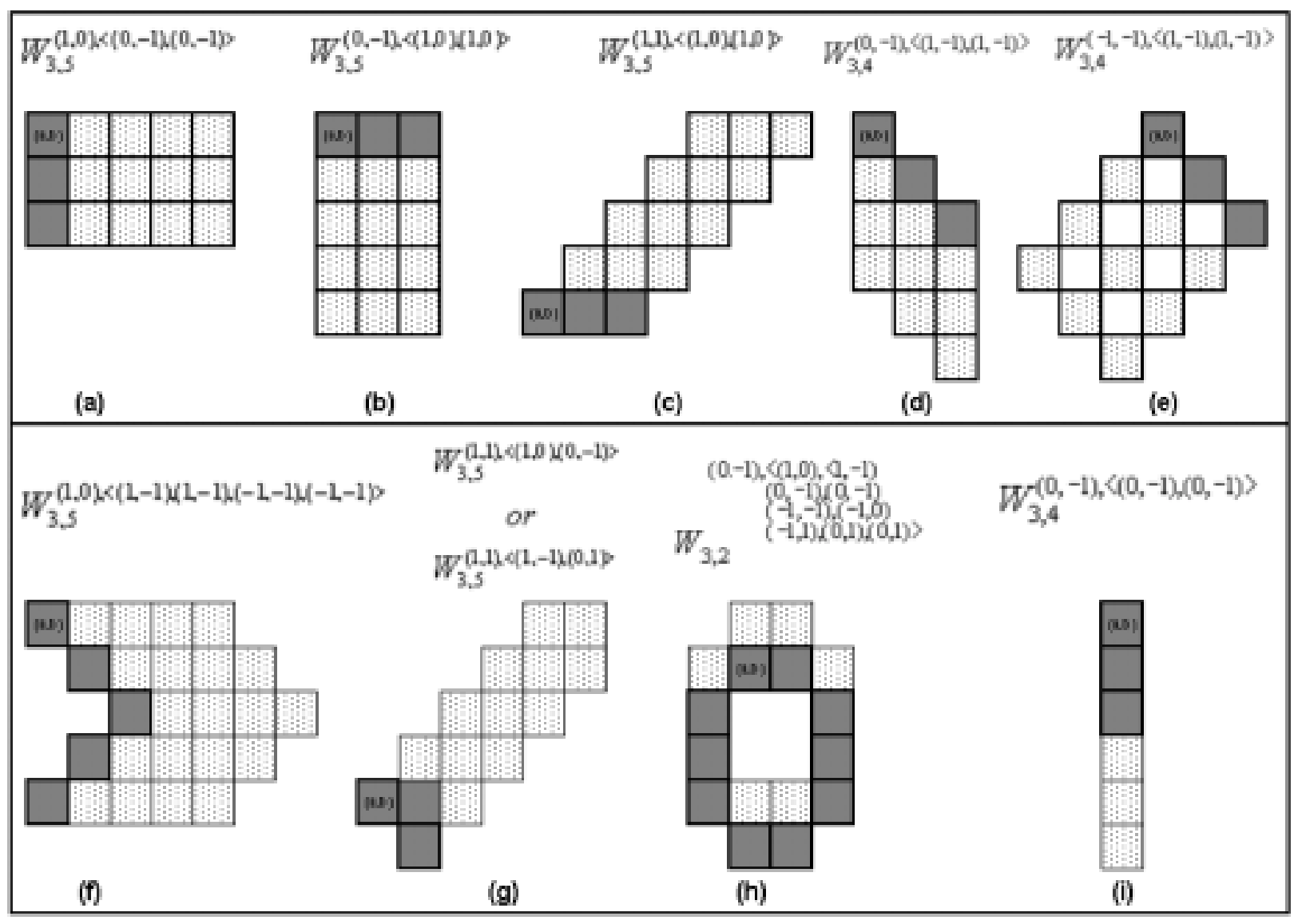

Figure 4. Some of the regular and irregular clique windows defined in $\eta^{2}$. (a),(b),(c),(d),(e) are regular, but (f),(g),(h),(i) are irregular. 


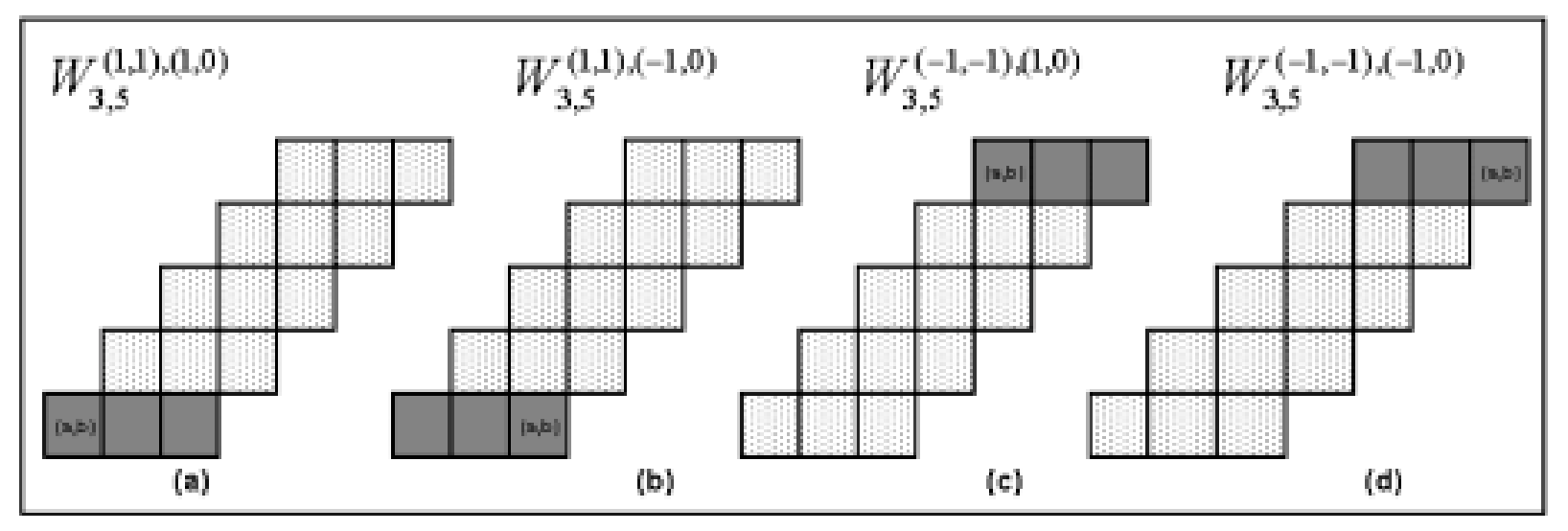

Figure 5. Different representations of a regular clique window.

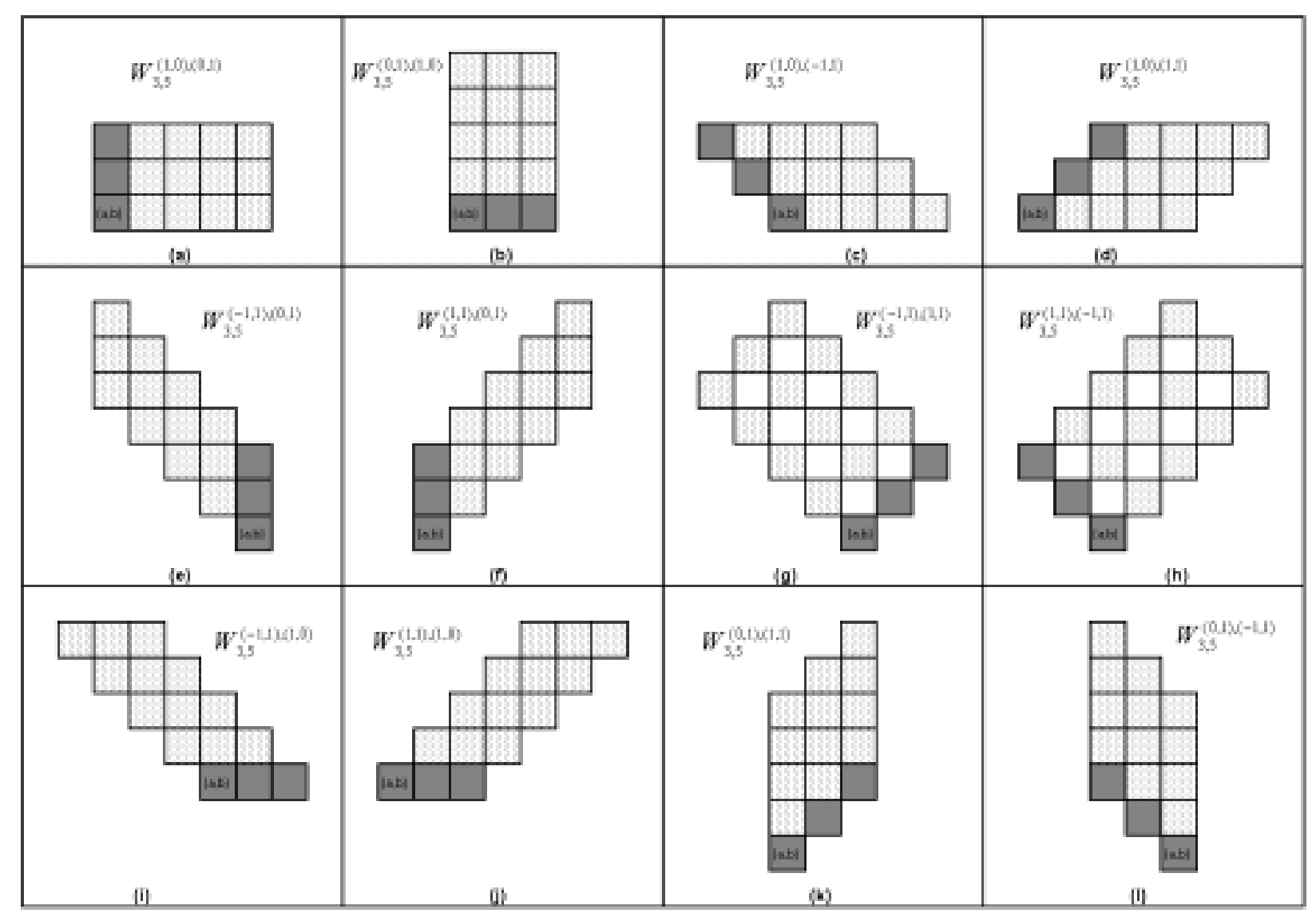

Figure 6. Regular clique windows defined in $\eta^{2}$. 


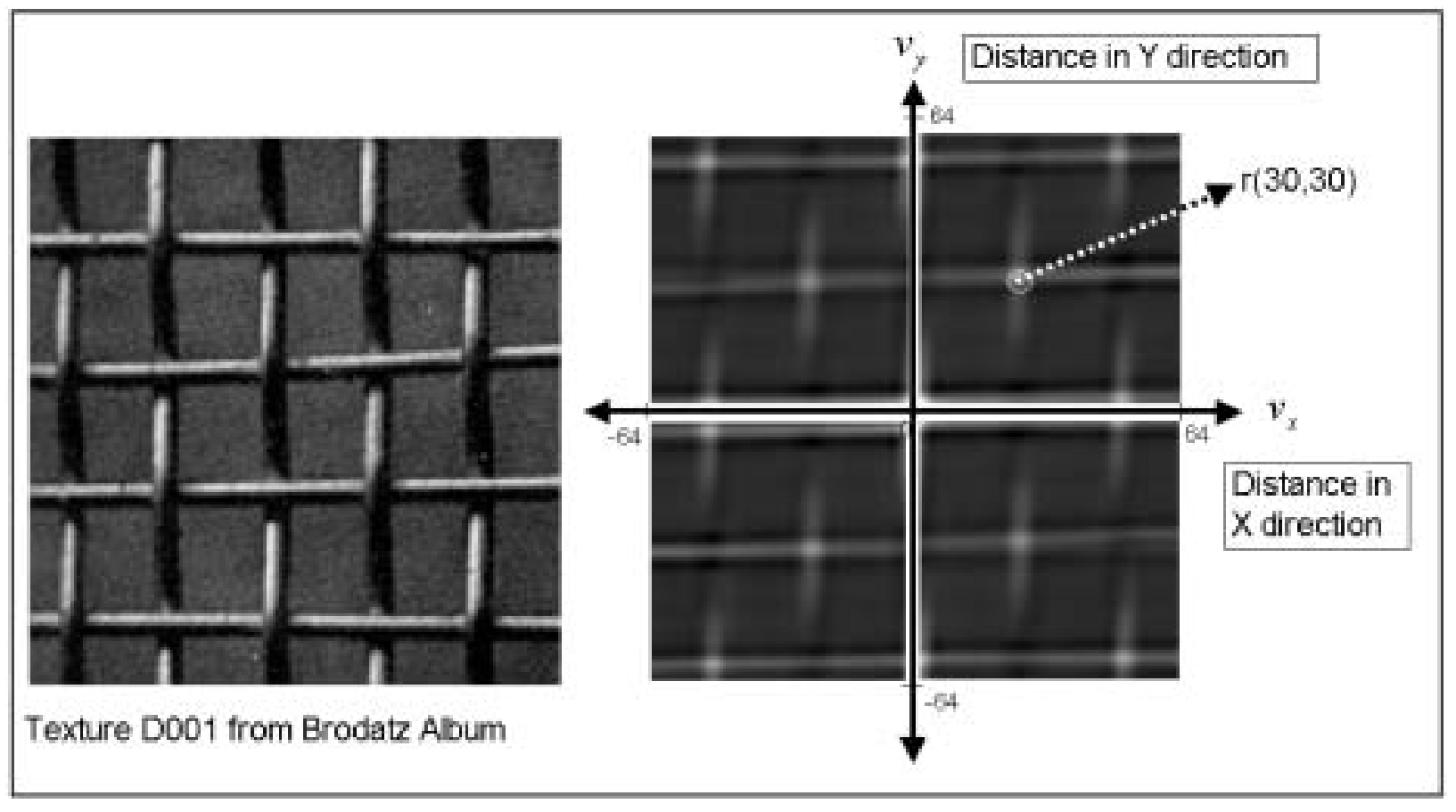

Figure 7: Texture D001 with size $128 \times 128$ and its correlogram as a 2-d intensity diagram.

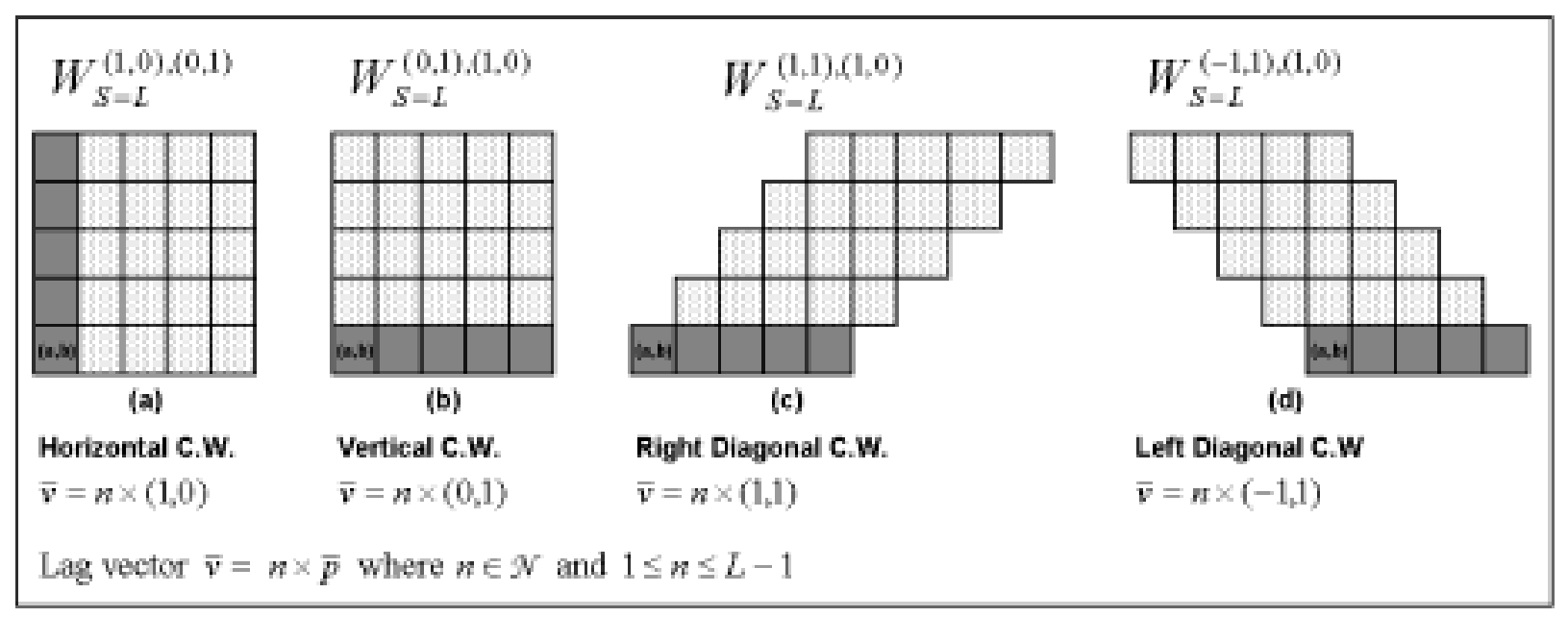

Figure 8. Horizontal, vertical, right and left diagonal clique windows. 

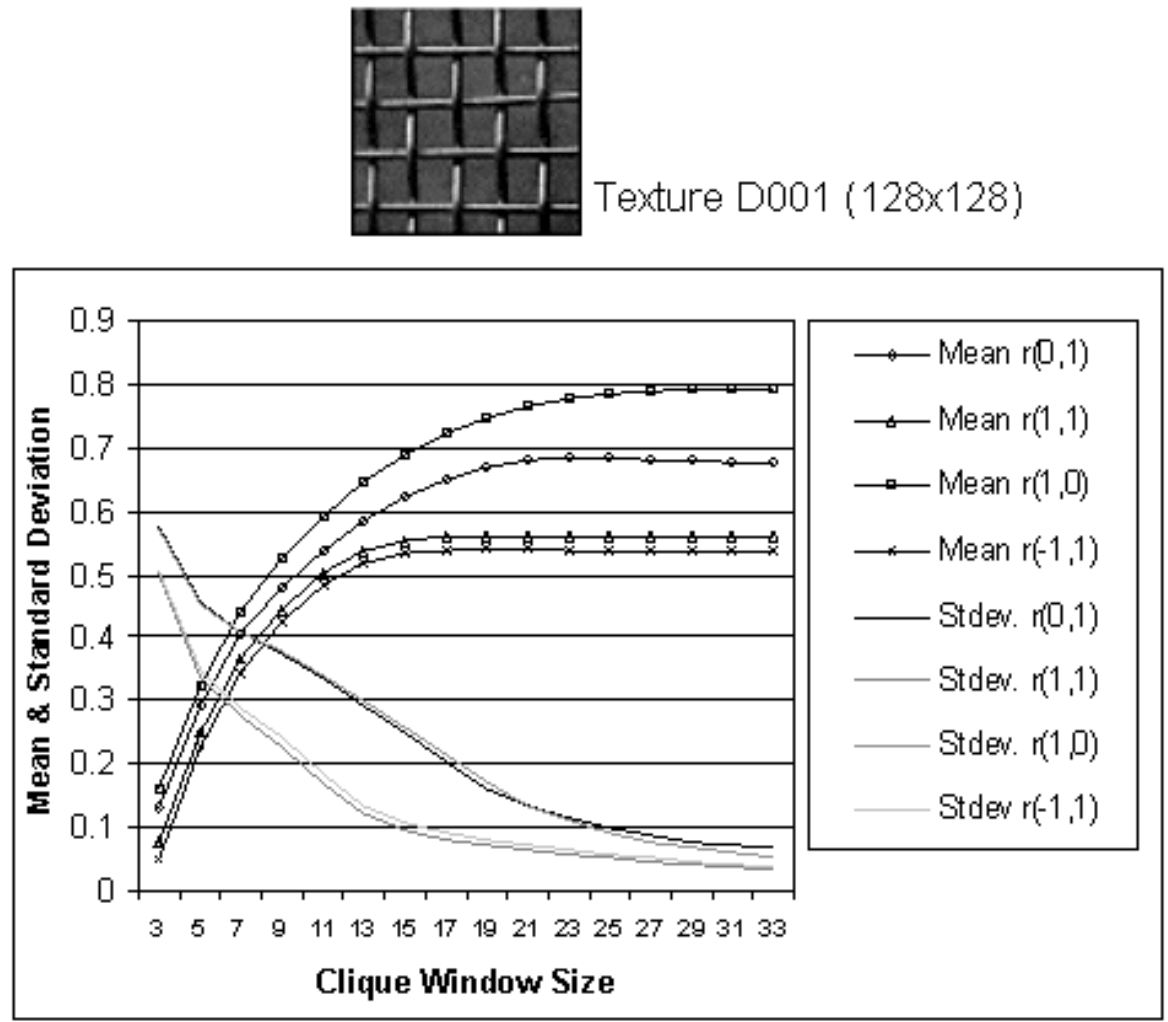

Figure 9. Window Sizes vs. Mean Values and Standard Deviations for texture D001 from Brodatz Album.

Texture D052 (128×128)

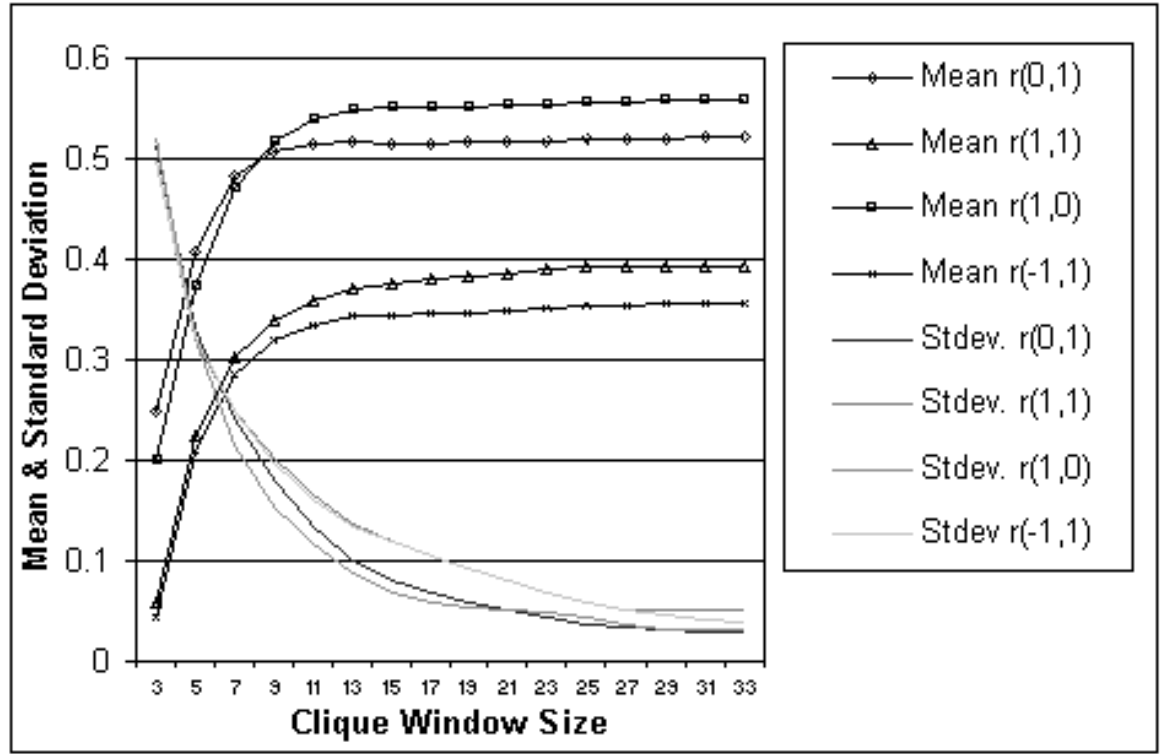

Figure 10. Window Sizes vs. Mean Values and Standard Deviations for texture D052 from Brodatz Album. 


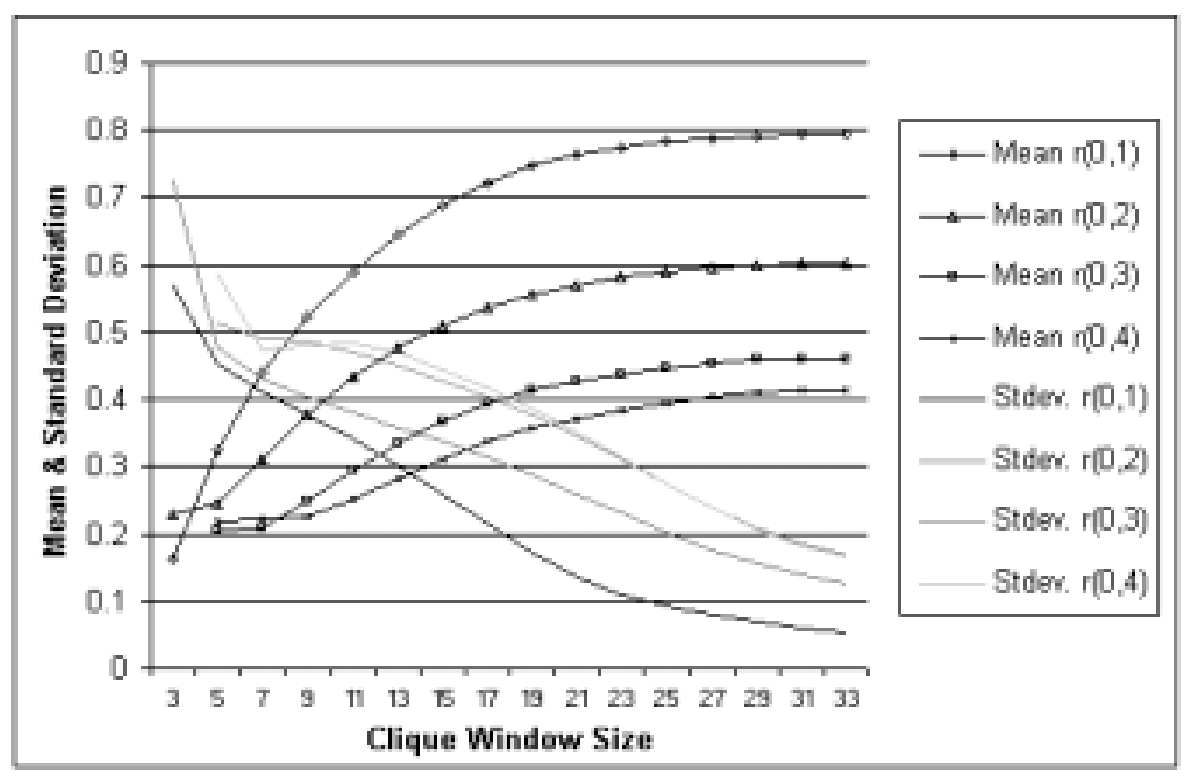

Figure 11. Window Sizes vs. Mean Values \& Standard Deviations for texture D001 using vertical clique windows.

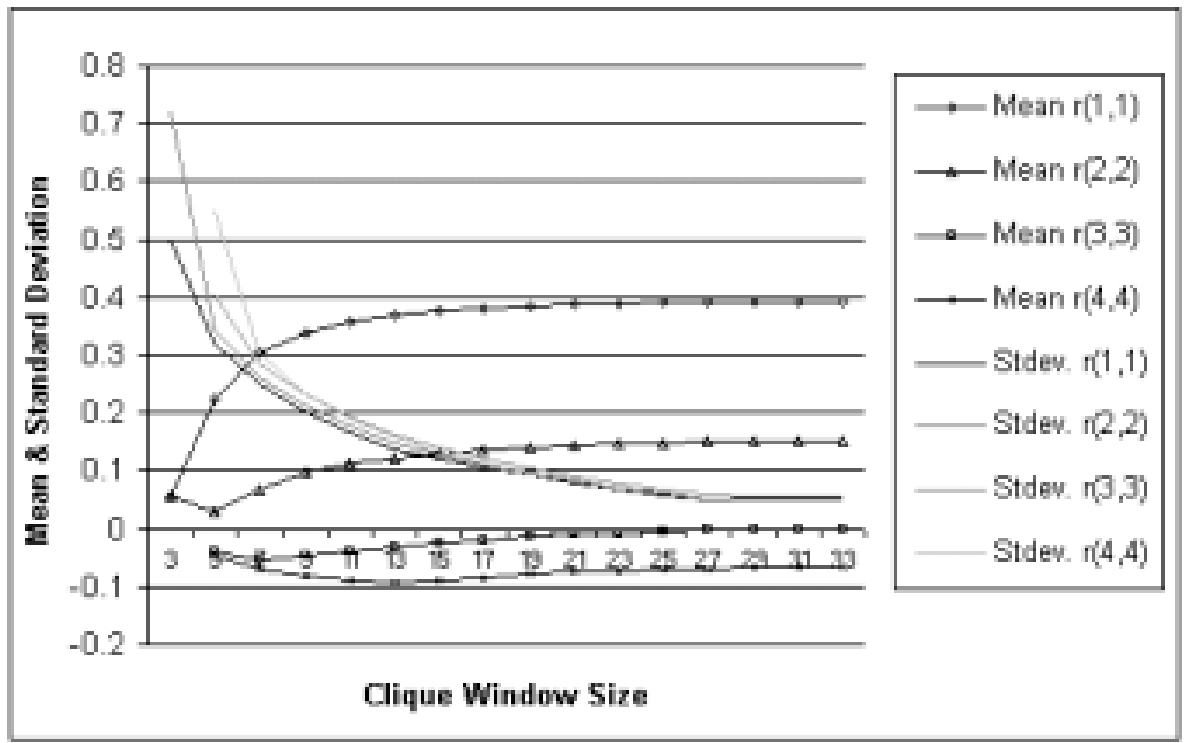

Figure 12. Window Sizes vs. Mean Values \& Standard Deviations for texture D052 using right diagonal clique windows. 


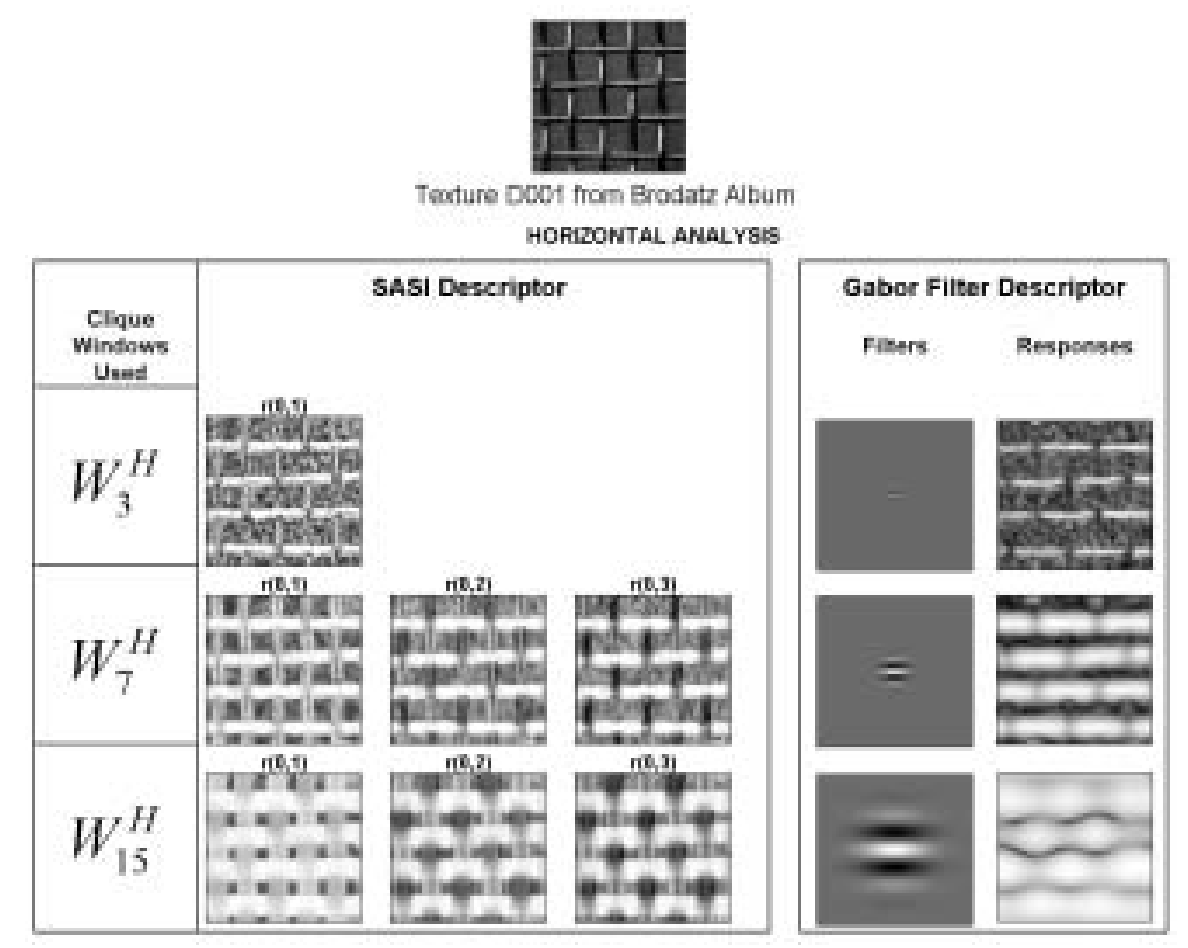

Figure 13. SASI and Gabor Filters horizontal analysis of texture D001 from Brodatz Album.

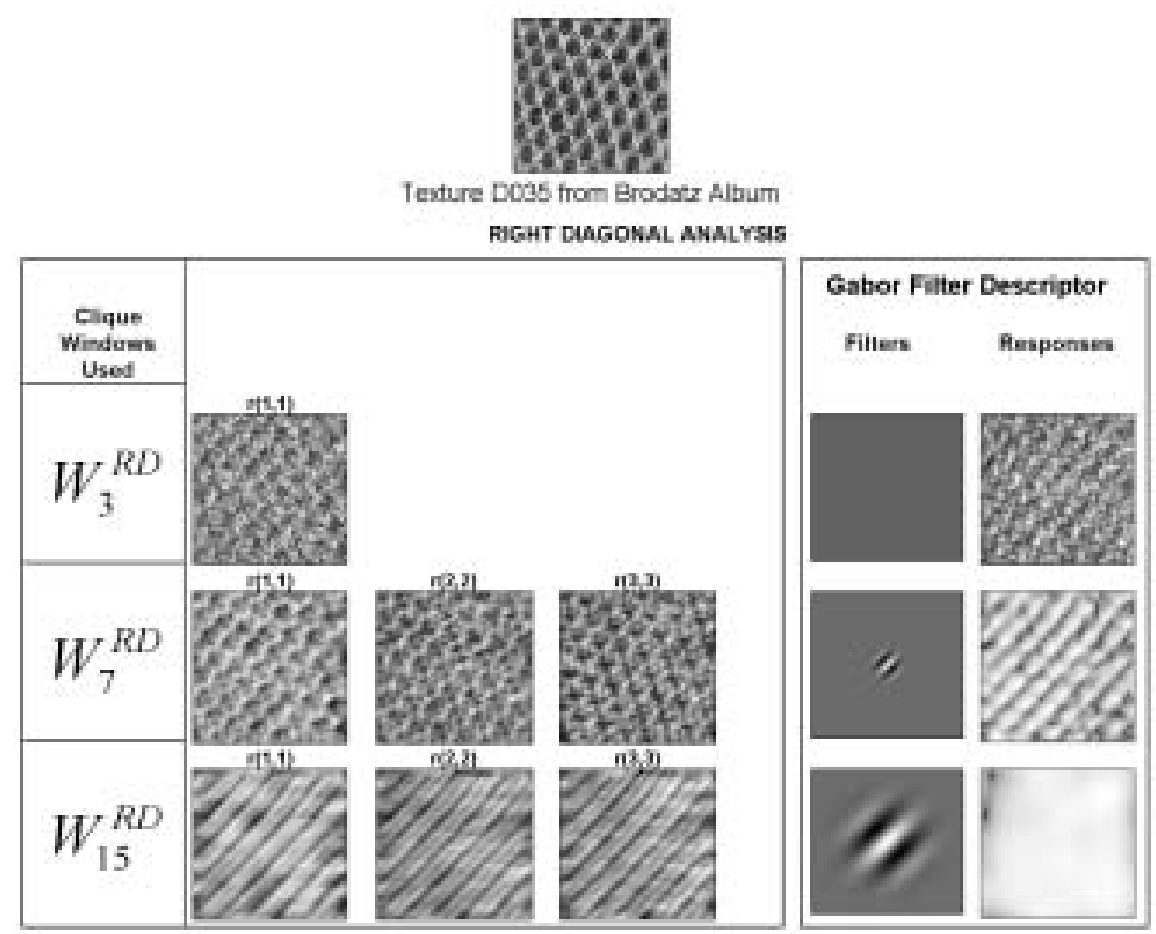

Figure 14. SASI and Gabor Filters right diagonal analysis of texture D035 from Brodatz Album. 


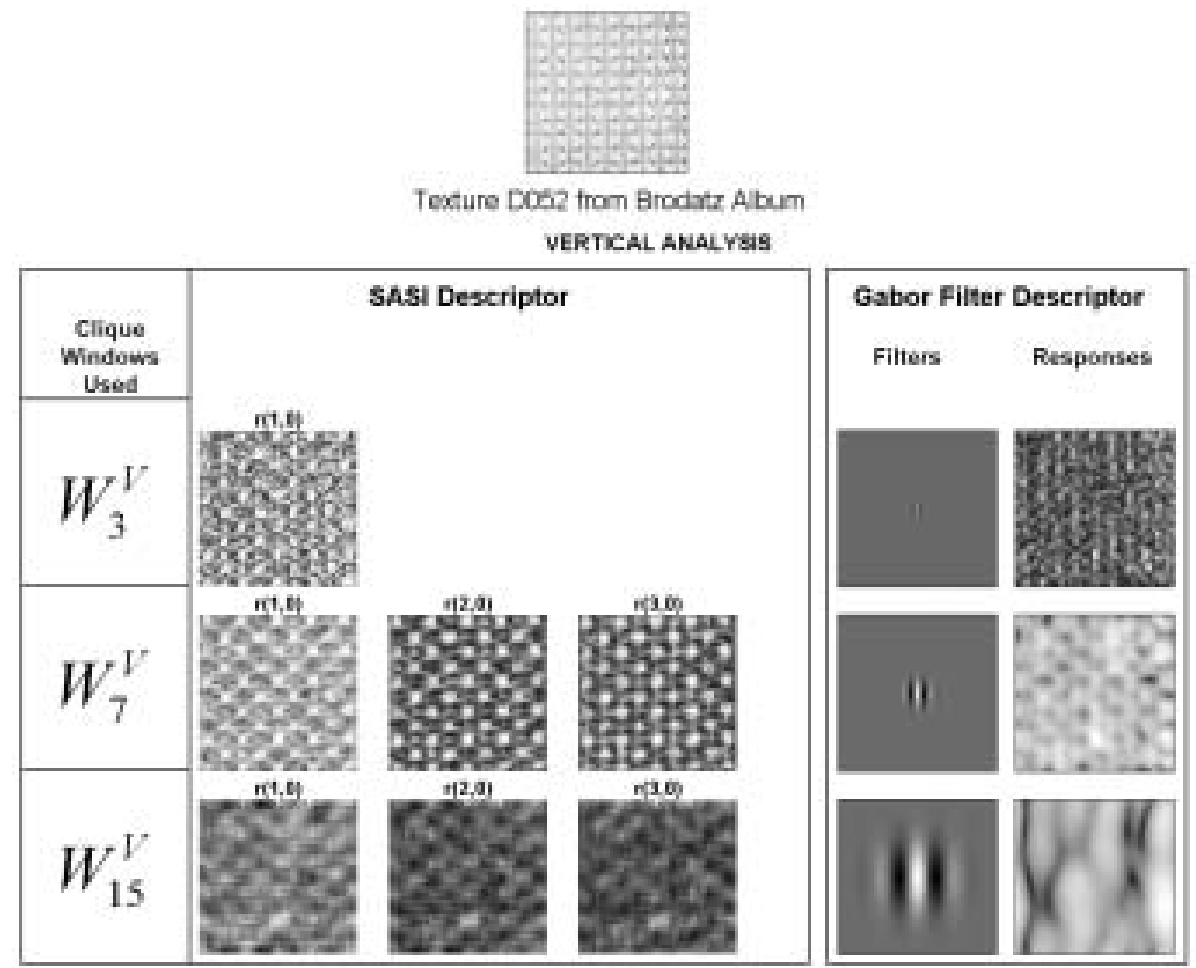

Figure 15. SASI and Gabor Filters vertical analysis of texture D052 from Brodatz Album.

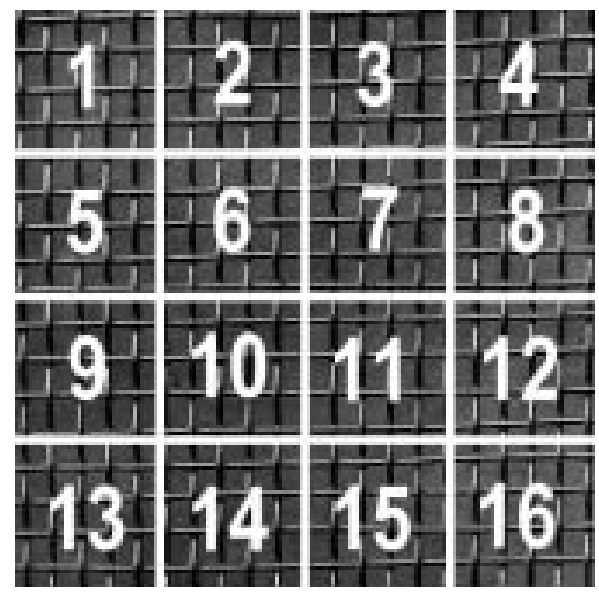

Figure 16: Subimages of size 128x128 in D001 with size 512x512. 


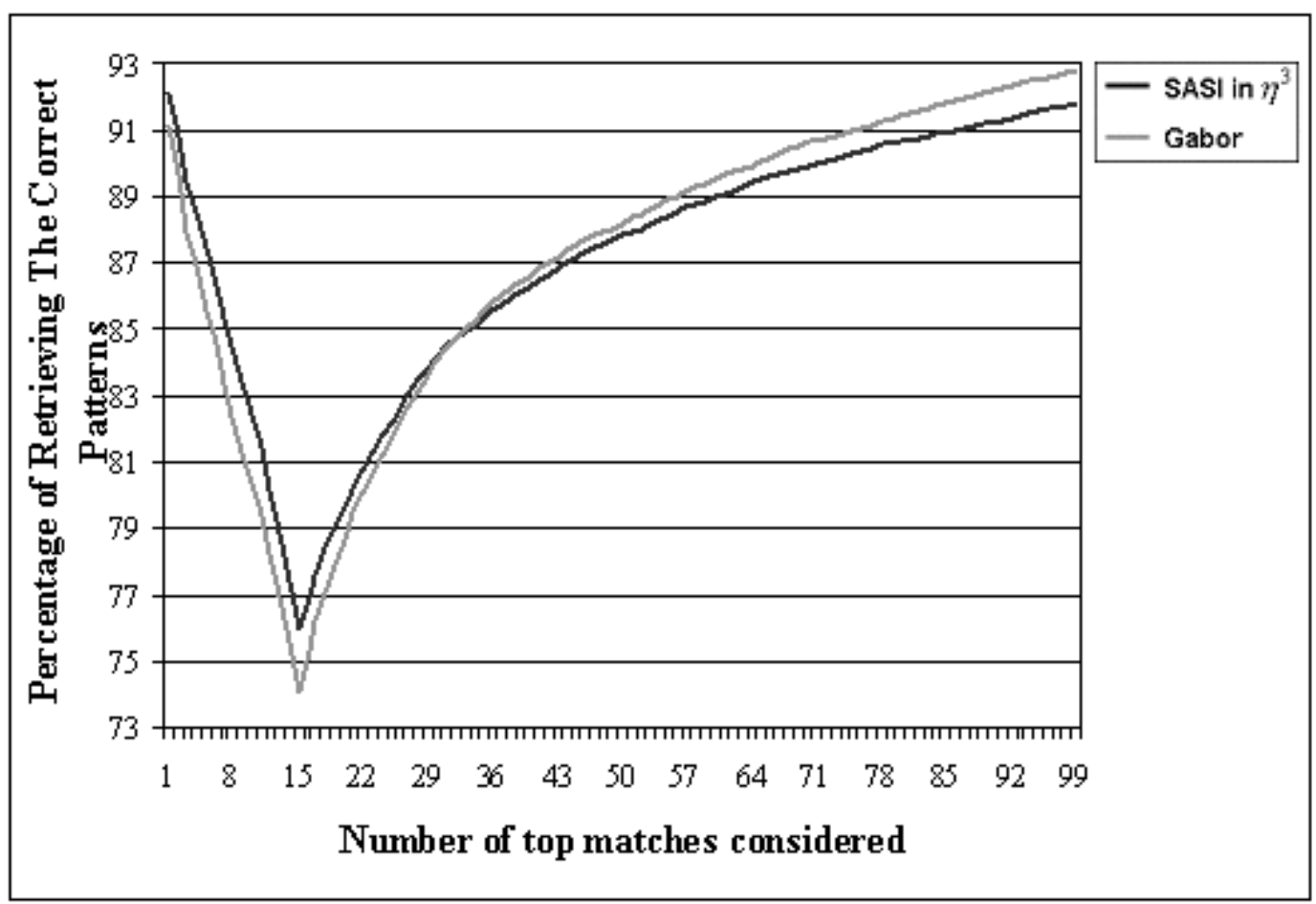

Figure 17. Average retrieval rates as a function of number of retrieved subimages for Brodatz Album.

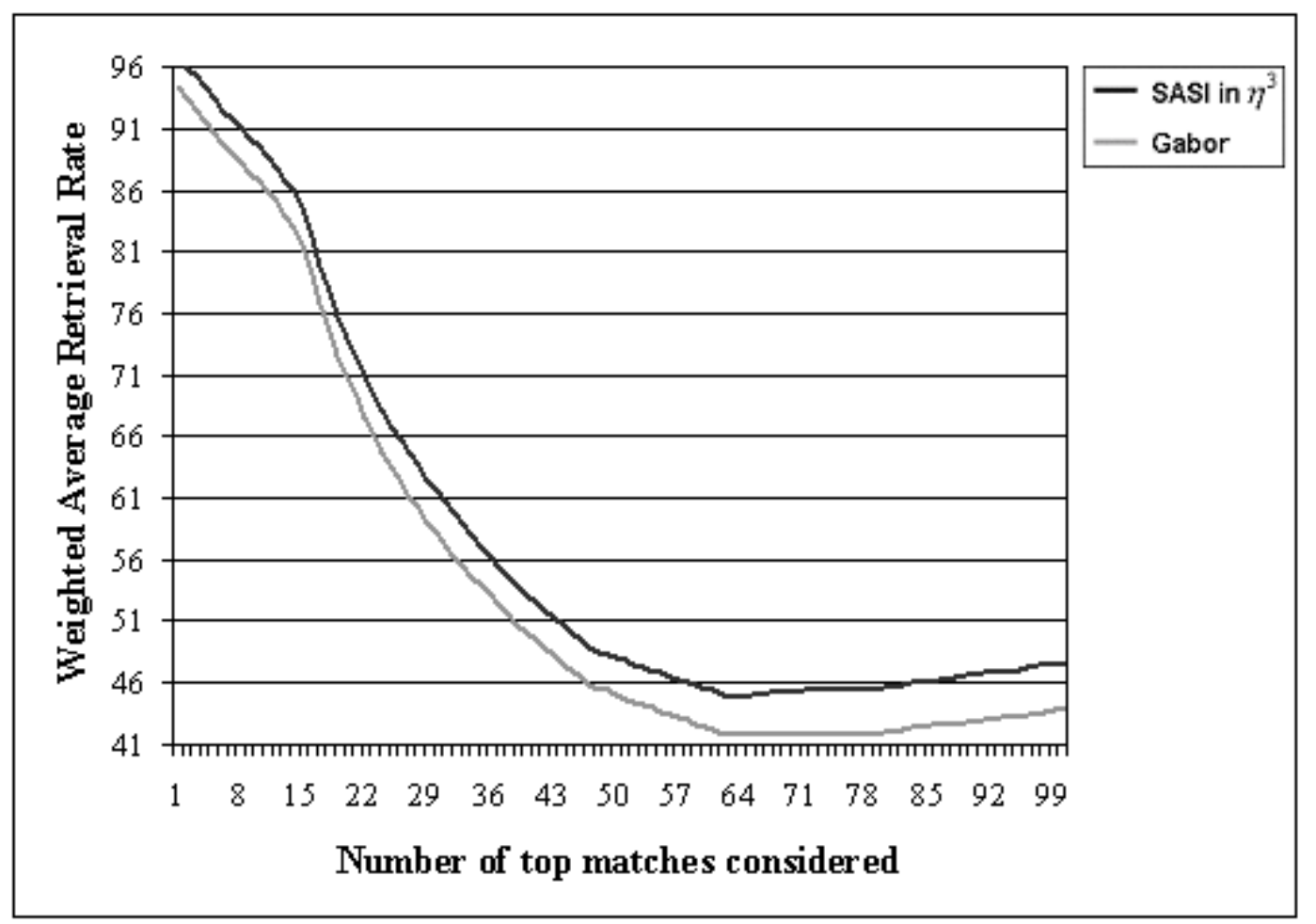

Figure 18. Retrieval performance after clustering for Brodatz Album. 


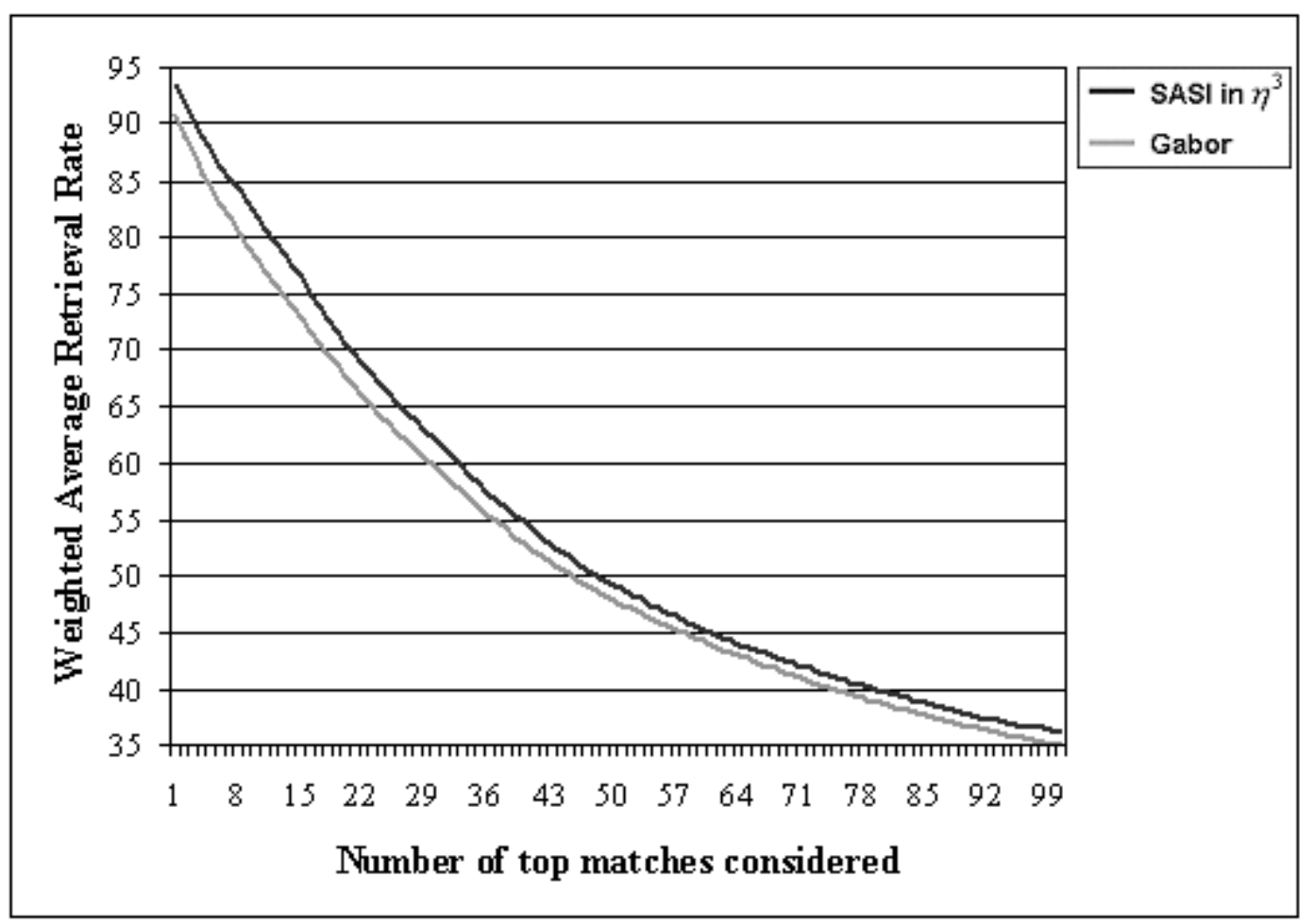

Figure 19. Retrieval performance after clustering for VisTex database.

\begin{tabular}{|c|c|c|c|}
\hline & \multicolumn{2}{|c|}{ 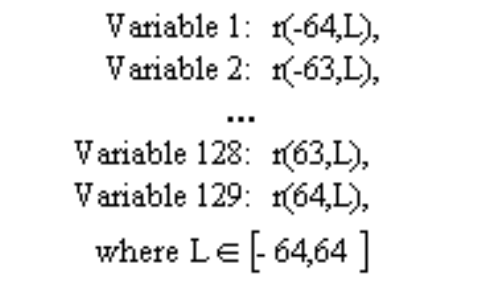 } & \\
\hline & Eigenvalue & Proportion & Cumulative \\
\hline Principal Component 1 & 89.415 & 0.693 & 0.693 \\
\hline Principal Component 2 & 16.753 & 0.130 & 0.823 \\
\hline Principal Component 3 & 13.180 & 0.102 & 0.925 \\
\hline Principal Component 4 & 4.626 & 0.036 & 0.961 \\
\hline Principal Component 5 & 2.395 & 0.019 & 0.980 \\
\hline Principal Component 6 & 0.883 & 0.007 & 0.986 \\
\hline Principal Component 7 & 0.570 & 0.004 & 0.991 \\
\hline Principal Component 8 & 0.341 & 0.003 & 0.994 \\
\hline Principal Component 9 & 0.205 & 0.002 & 0.995 \\
\hline Principal Component 10 & 0.198 & 0.002 & 0.997 \\
\hline$\ldots$ & $\ldots$ & $\ldots$ & $\ldots$ \\
\hline Principal Component 129 & 0 & 0 & 1 \\
\hline
\end{tabular}

Table 1: Principal Component Analysis of the correlogram. 


\begin{tabular}{|c|c|}
\hline SASI Clique Window Size & Gabor Filter Parameters \\
\cline { 1 - 2 } $3 \times 3$ & $\begin{array}{c}\text { Lower Frequency }=0.05 \\
\text { Higher Frequency }=0.4 \\
\text { Scale }=3, \text { Onientation }=4\end{array}$ \\
\hline $7 \times 7$ &
\end{tabular}

Table 2: Parameters of Gabor and SASI.

\begin{tabular}{|c|c|c|c|c|c|c|}
\hline & & Brodutz. & $\begin{array}{c}\text { CUReT } \\
\text { Texture } \\
\text { Datalbase }\end{array}$ & $\begin{array}{c}\text { Photemetric } \\
\text { Texture } \\
\text { Datalase }\end{array}$ & VisTex & $\begin{array}{l}\text { New Imsage } \\
\text { Database }\end{array}$ \\
\hline \multicolumn{2}{|l|}{ Properties } & $\begin{array}{c}512 \times 512 \\
\text { Gray valued }\end{array}$ & $\begin{array}{c}\text { Various size, } \\
\text { Colored }\end{array}$ & $\begin{array}{c}512 \times 512 \\
\text { Gray valued }\end{array}$ & $\begin{array}{l}512 \times 512 \\
\text { Colored }\end{array}$ & $\begin{array}{c}\text { Various Size, } \\
\text { Mixed }\end{array}$ \\
\hline \multicolumn{2}{|l|}{ Preprocessing } & - & $\begin{array}{c}\text { Gray Scaled, } \\
\text { Rescaled }\end{array}$ & - & Gray Scaled & $\begin{array}{l}\text { Gray Scaled, } \\
\text { Rescaled }\end{array}$ \\
\hline \multicolumn{2}{|l|}{ Total if of Images in the Database } & 112 & 61 & 30 & 167 & 370 \\
\hline \multicolumn{2}{|l|}{ \# of Subimage8 } & 1,792 & 976 & 480 & 2,672 & 5,920 \\
\hline \multicolumn{2}{|l|}{ GABOR - Average Retrieval Rate } & 74.0746 & $78.61 \%$ & 80.2746 & $46.19 \% \mathrm{~b}$ & $60.56 \%$ \\
\hline \multirow{5}{*}{ SASI - Average Retrieval Rate } & in $\eta^{1}$ & $70.40 \%$ & $83.82 \%$ & $86.84 \%$ & $47.86 \%$ & $62.71 \%$ \\
\hline & in $\eta^{2}$ & $75.47 \%$ & $85.38 \%$ & $90.75 \%$ & $51.80 \%$ & $66.71 \%$ \\
\hline & in $\eta^{3}$ & $75.93 \% 6$ & $85.68 \%$ & 92.7046 & $52.54 \%$ & $67.20 \%$ \\
\hline & in $\eta^{4}$ & $75.31 \%$ & $83.75 \%$ & $92.27 \%$ & $51.51 \%$ & $66.10 \%$ \\
\hline & in $\eta^{5}$ & $74.84 \%$ & $82.21 \%$ & $92.11 \%$ & $50.65 \%$ & $65.29 \%$ \\
\hline
\end{tabular}

Table 3: Properties of the Brodatz, CUReT, PhoTex and VisTex image databases and corresponding average retrieval rates. 


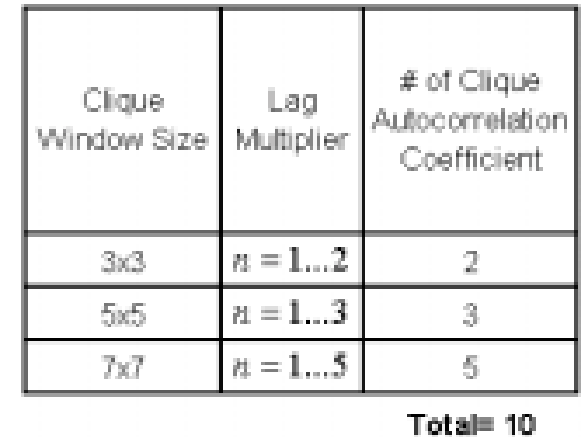

(a)

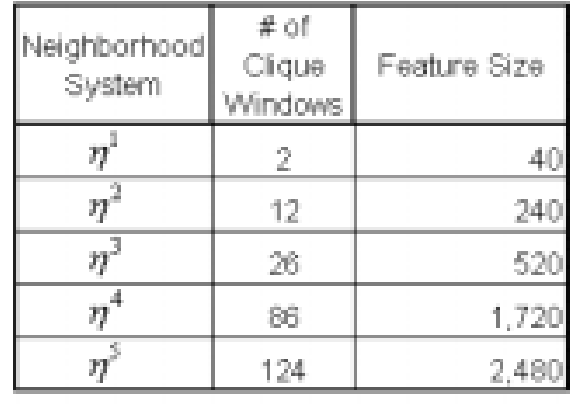

(b)

Table 4: The clique window sizes and autocorrelation coefficients (a) and number of clique windows vs. feature size (b) for Brodatz Album, CUReT, PhoTex and VisTex image databases.

\begin{tabular}{|l|l|l|}
\hline Texture & Gabor & SASI \\
\hline D001 & 99.17 & 100.00 \\
\hline D002 & 74.58 & 61.25 \\
\hline D003 & 91.67 & 82.08 \\
\hline D004 & 100.00 & 90.00 \\
\hline D005 & 68.75 & 58.33 \\
\hline D006 & 100.00 & 100.00 \\
\hline D007 & 51.25 & 49.58 \\
\hline D008 & 90.83 & 96.25 \\
\hline D009 & 95.42 & 92.50 \\
\hline D010 & 80.00 & 79.58 \\
\hline D011 & 100.00 & 100.00 \\
\hline D012 & 89.17 & 90.42 \\
\hline D013 & 62.92 & 52.08 \\
\hline D014 & 100.00 & 100.00 \\
\hline D015 & 65.00 & 65.00 \\
\hline D016 & 100.00 & 100.00 \\
\hline D017 & 100.00 & 100.00 \\
\hline D018 & 86.67 & 95.83 \\
\hline D019 & 86.67 & 99.58 \\
\hline D020 & 100.00 & 100.00 \\
\hline D021 & 100.00 & 100.00 \\
\hline D022 & 71.25 & 87.08 \\
\hline D023 & 55.83 & 44.17 \\
\hline D024 & 93.75 & 93.75 \\
\hline D025 & 97.92 & 81.25 \\
\hline D026 & 80.83 & 80.83 \\
\hline D027 & 53.33 & 34.17 \\
\hline D028 & 82.92 & 81.67 \\
\hline D029 & 97.92 & 100.00 \\
\hline D030 & 40.83 & 50.00 \\
\hline D031 & 24.58 & 39.58 \\
\hline D032 & 99.17 & 97.92 \\
\hline D033 & 90.42 & 82.08 \\
\hline D034 & 95.42 & 96.25 \\
\hline D035 & 80.42 & 80.42 \\
\hline D036 & 66.67 & 72.50 \\
\hline D037 & 99.17 & 100.00 \\
\hline D038 & 85.83 & 81.67 \\
\hline & & \\
\hline
\end{tabular}

\begin{tabular}{|l|l|l|}
\hline Texture & Gabor & SASI \\
\hline D039 & 52.08 & 67.92 \\
\hline D040 & 70.42 & 75.42 \\
\hline D041 & 71.67 & 73.33 \\
\hline D042 & 33.75 & 50.00 \\
\hline D043 & 10.00 & 12.08 \\
\hline D044 & 12.92 & 15.42 \\
\hline D045 & 11.67 & 22.08 \\
\hline D046 & 89.17 & 95.42 \\
\hline D047 & 100.00 & 100.00 \\
\hline D048 & 72.92 & 97.08 \\
\hline D049 & 100.00 & 100.00 \\
\hline D050 & 76.25 & 80.42 \\
\hline D051 & 79.58 & 85.83 \\
\hline D052 & 67.08 & 100.00 \\
\hline D053 & 100.00 & 100.00 \\
\hline D054 & 48.75 & 53.75 \\
\hline D055 & 100.00 & 100.00 \\
\hline D056 & 100.00 & 100.00 \\
\hline D057 & 100.00 & 100.00 \\
\hline D058 & 15.42 & 15.42 \\
\hline D059 & 27.92 & 22.08 \\
\hline D060 & 64.17 & 42.92 \\
\hline D061 & 44.17 & 38.75 \\
\hline D062 & 71.67 & 65.00 \\
\hline D063 & 30.83 & 23.75 \\
\hline D064 & 99.17 & 100.00 \\
\hline D065 & 100.00 & 100.00 \\
\hline D066 & 90.00 & 95.83 \\
\hline D067 & 62.92 & 63.75 \\
\hline D068 & 98.33 & 100.00 \\
\hline D069 & 48.75 & 33.75 \\
\hline D070 & 50.00 & 81.25 \\
\hline D071 & 61.67 & 72.50 \\
\hline D072 & 57.92 & 54.17 \\
\hline D073 & 42.92 & 60.00 \\
\hline D074 & 89.17 & 79.17 \\
\hline D075 & 97.92 & 100.00 \\
\hline D076 & 99.17 & 100.00 \\
\hline
\end{tabular}

\begin{tabular}{|l|l|l|}
\hline Texture & Gabor & SASI \\
\hline D077 & 100.00 & 100.00 \\
\hline D078 & 97.08 & 97.08 \\
\hline D079 & 96.67 & 100.00 \\
\hline D080 & 86.25 & 74.58 \\
\hline D081 & 100.00 & 97.92 \\
\hline D082 & 100.00 & 100.00 \\
\hline D083 & 99.58 & 100.00 \\
\hline D084 & 100.00 & 100.00 \\
\hline D085 & 100.00 & 100.00 \\
\hline D086 & 68.75 & 82.50 \\
\hline D087 & 94.58 & 100.00 \\
\hline D088 & 22.08 & 64.58 \\
\hline D089 & 34.58 & 63.33 \\
\hline D090 & 37.50 & 37.92 \\
\hline D091 & 34.58 & 22.50 \\
\hline D092 & 96.25 & 96.25 \\
\hline D093 & 87.50 & 75.42 \\
\hline D094 & 99.58 & 88.75 \\
\hline D095 & 100.00 & 99.58 \\
\hline D096 & 77.08 & 82.92 \\
\hline D097 & 34.17 & 26.25 \\
\hline D098 & 42.50 & 57.08 \\
\hline D099 & 37.08 & 40.83 \\
\hline D100 & 41.67 & 45.00 \\
\hline D101 & 57.50 & 98.75 \\
\hline D102 & 74.17 & 99.17 \\
\hline D103 & 66.67 & 75.83 \\
\hline D104 & 68.33 & 90.42 \\
\hline D105 & 67.50 & 50.00 \\
\hline D106 & 58.33 & 46.67 \\
\hline D107 & 52.92 & 35.83 \\
\hline D108 & 45.83 & 36.25 \\
\hline D109 & 79.58 & 94.58 \\
\hline D110 & 98.75 & 93.75 \\
\hline D111 & 60.83 & 82.08 \\
\hline D112 & 71.67 & 55.42 \\
\hline & & \\
\hline Average 74.07 & 75.93 \\
\hline
\end{tabular}

Table 5: The retrieval rates for the 112 texture images in Brodatz Album. 


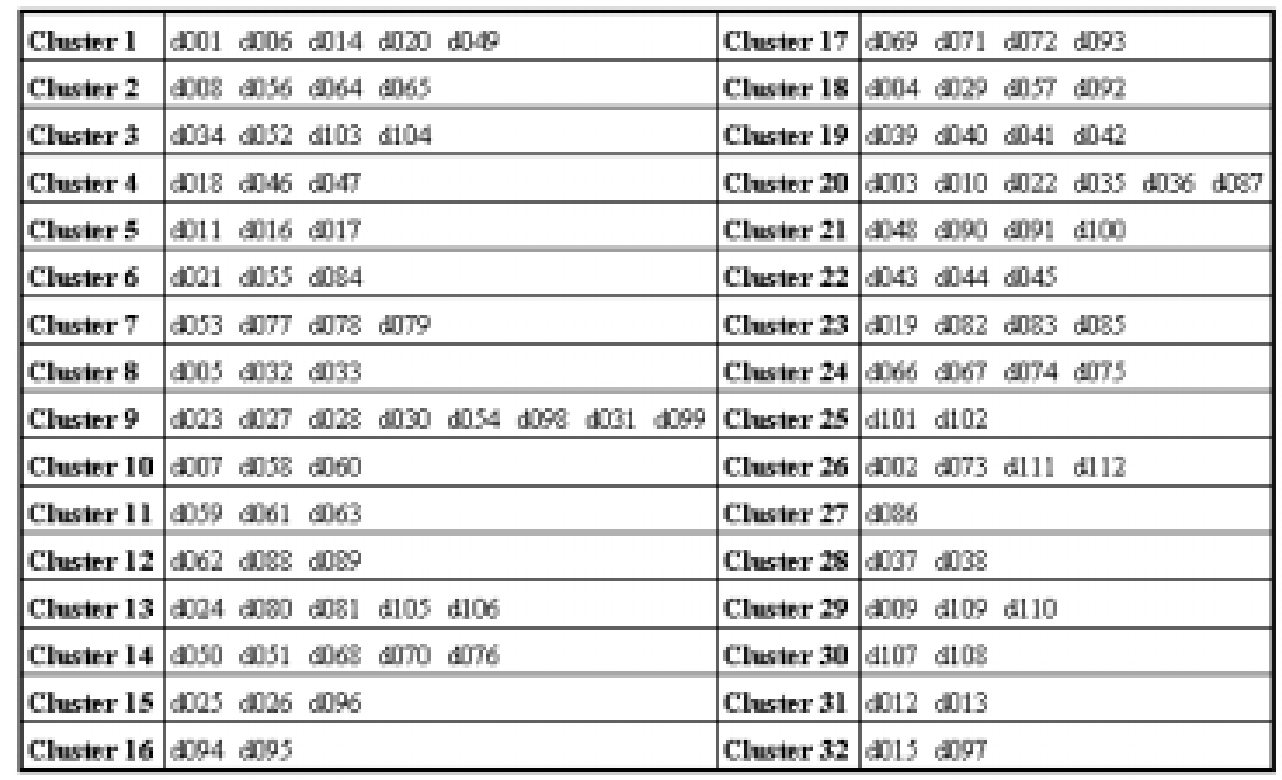

Table 6: Texture clusters identified by human for Brodatz Album.

\begin{tabular}{|l|l|r|}
\hline No & Grou Name & $\begin{array}{l}\text { Number } \\
\text { of Image }\end{array}$ \\
\hline $\mathbf{1}$ & Bark & 13 \\
\hline $\mathbf{2}$ & Brick & 9 \\
\hline $\mathbf{3}$ & Building & 11 \\
\hline $\mathbf{4}$ & Cloud & 2 \\
\hline $\mathbf{5}$ & Fabric & 20 \\
\hline $\mathbf{6}$ & Flower & 8 \\
\hline $\mathbf{7}$ & Food & 12 \\
\hline $\mathbf{8}$ & Grass & 3 \\
\hline $\mathbf{9}$ & Leaves & 17 \\
\hline
\end{tabular}

\begin{tabular}{|l|l|r|}
\hline No & Grou Name & $\begin{array}{r}\text { Number } \\
\text { of Image }\end{array}$ \\
\hline $\mathbf{1 0}$ & Metal & 6 \\
\hline $\mathbf{1 1}$ & Misc. & 4 \\
\hline $\mathbf{1 2}$ & Paintings & 13 \\
\hline $\mathbf{1 3}$ & Sand & 7 \\
\hline $\mathbf{1 4}$ & Stone & 6 \\
\hline $\mathbf{1 5}$ & Terrain & 11 \\
\hline $\mathbf{1 6}$ & Tile & 11 \\
\hline $\mathbf{1 7}$ & Water & 8 \\
\hline $\mathbf{1 8}$ & WheresWaldo & 3 \\
\hline $\mathbf{1 9}$ & Wood & 3 \\
\hline
\end{tabular}

Table 7: Image groups in the VisTex database. 


\section{Query Image: D001_1}

Gabor Descriptor Retrieval Results

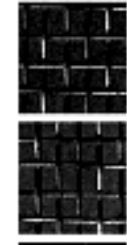

$5 \frac{1}{1}$
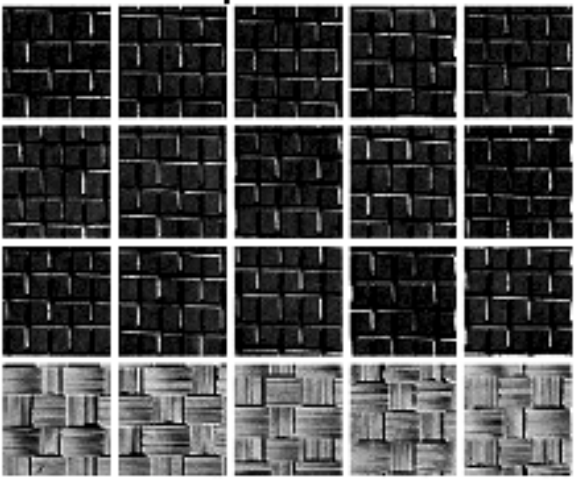

ill
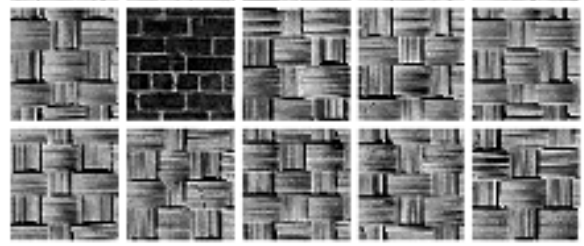

ih

ज्ञात
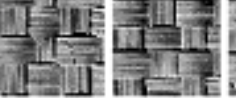

iा

IIIIIIIIIII
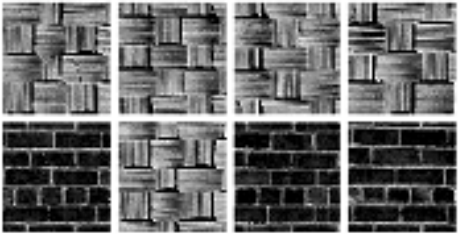

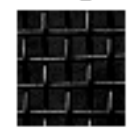

SASI Descriptor Retrieval Results
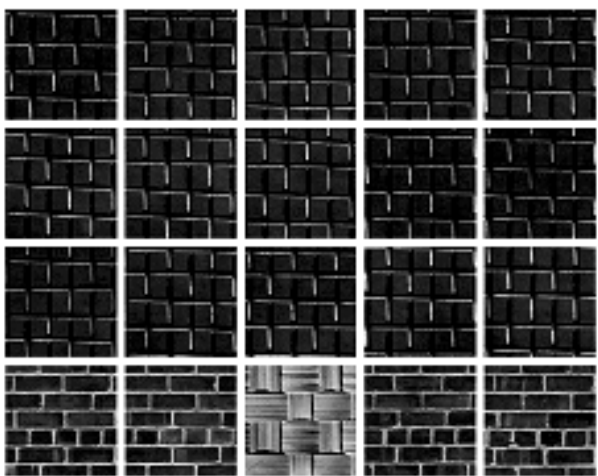

$\int_{1}^{1}$
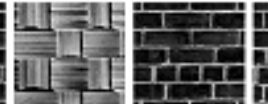

$\frac{1}{11}$
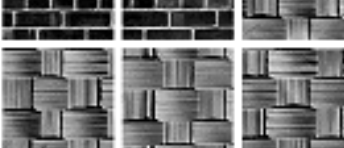

लिiा

एक्षे
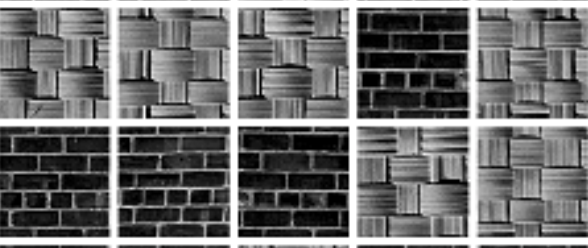

\begin{tabular}{c|}
\hline 1 \\
$\frac{1}{1}$ \\
\hline$x$
\end{tabular}
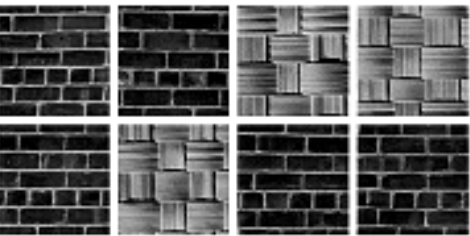

Query Image: D018_1

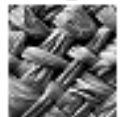

Gabor Descriptor Retrieval Results

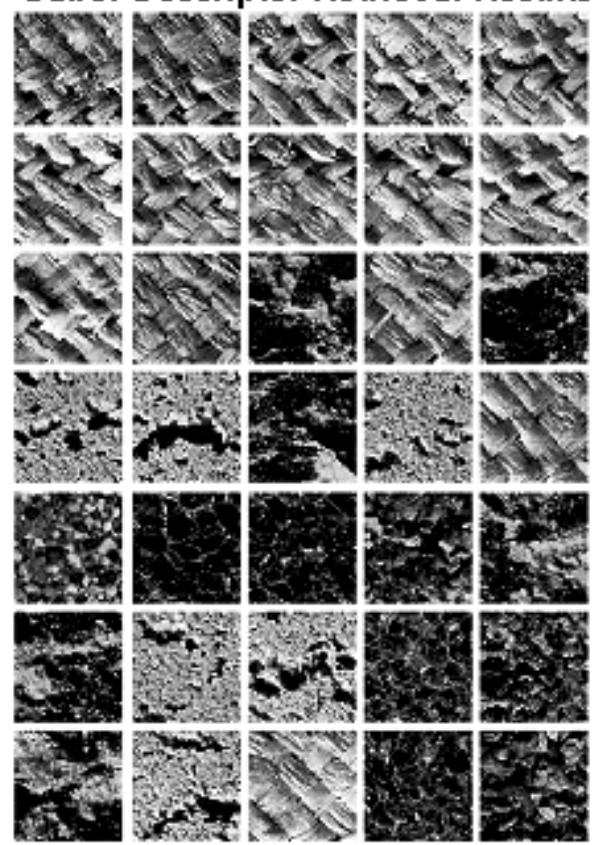

SASI Descriptor Retrieval Results

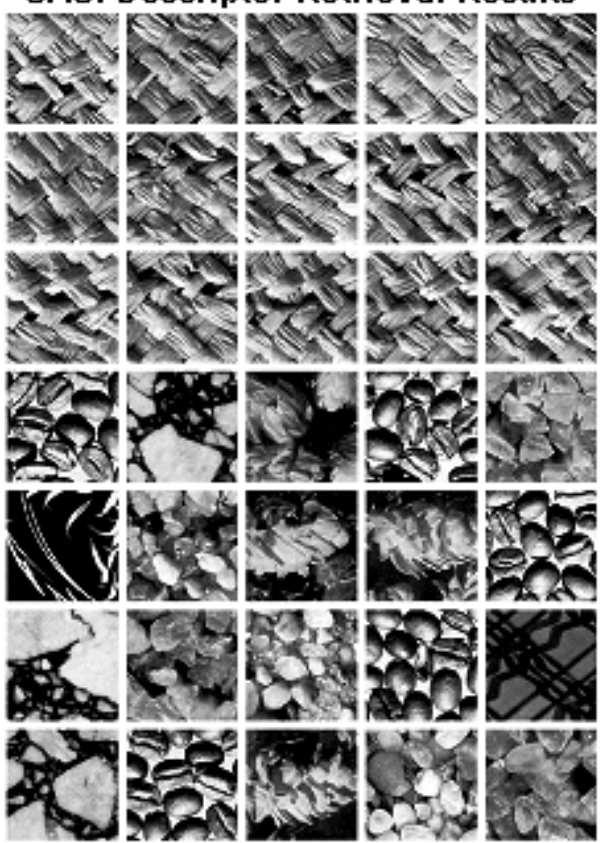

Appendix-Figure I.a: The most similar 35 images of given query images, namely D001_1 and D018_1,are depicted. Images are ordered by the distance from left to right, top to bottom (excluding self matches). 
Query Image: D052_1

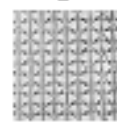

Gabor Descriptor Retrieval Results

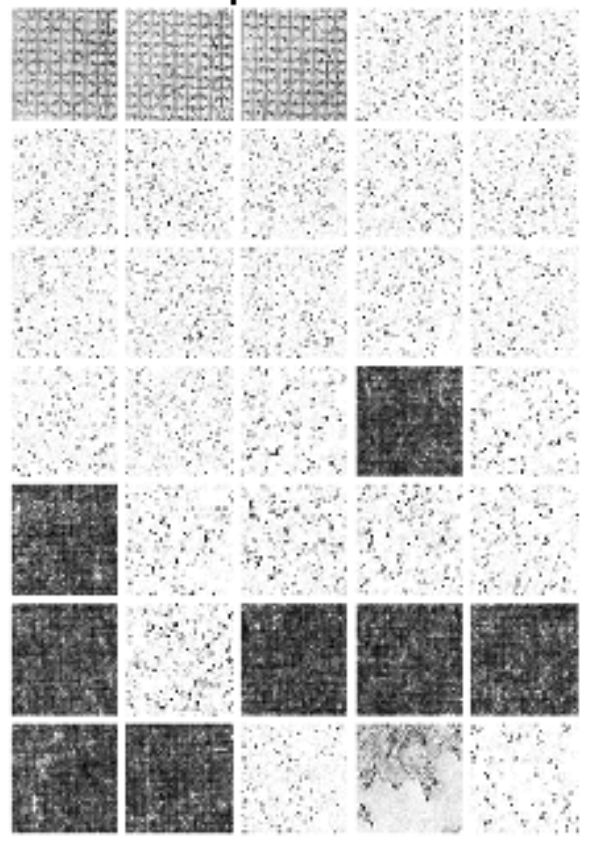

SASI Descriptor Retrieval Results

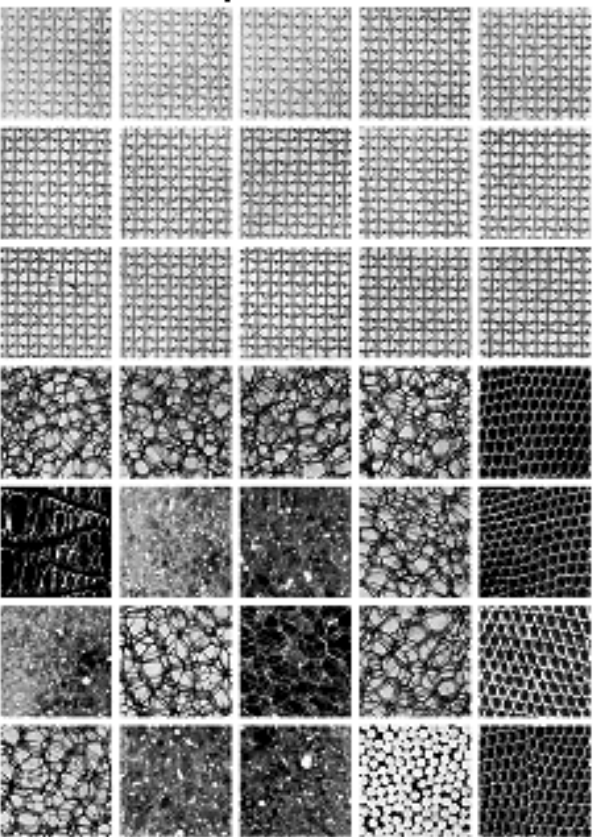

Query Image: D101_1

8.

Gabor Descriptor Retrieval Results

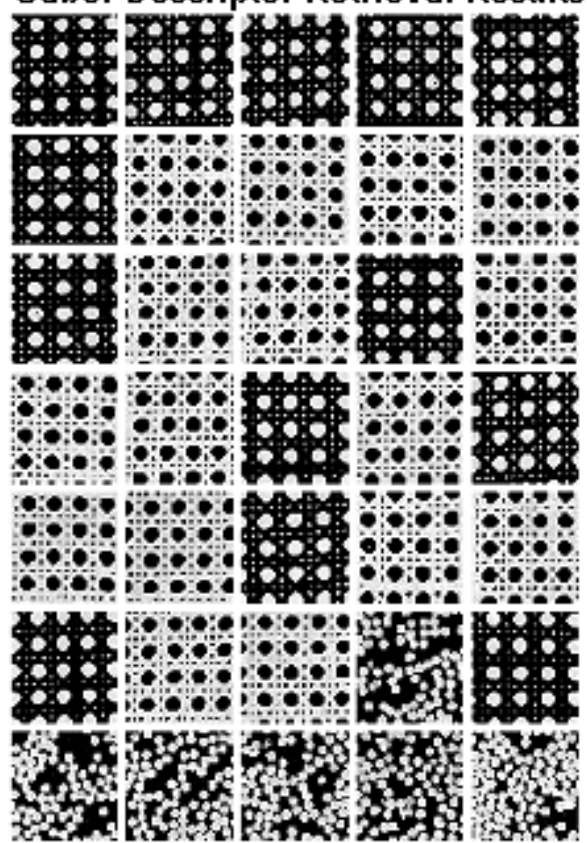

SASI Descriptor Retrieval Results

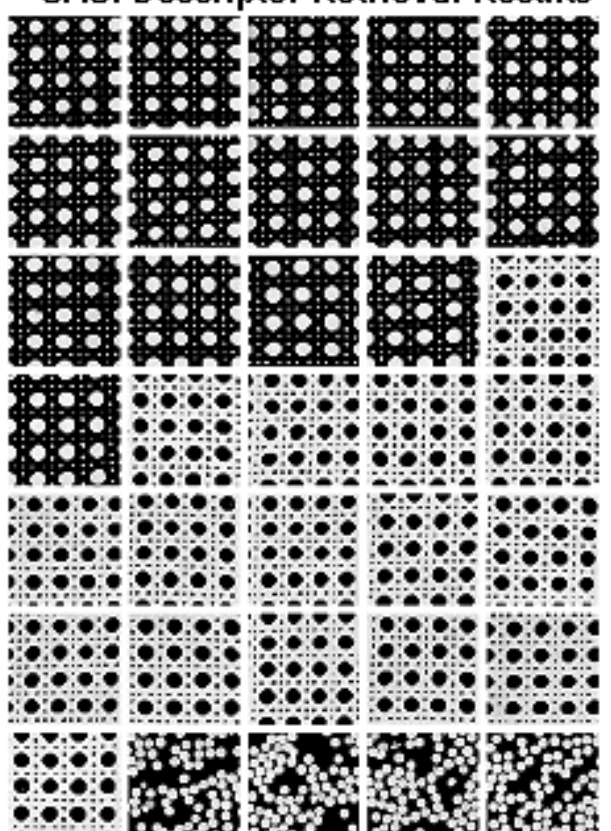

Appendix-Figure I.b: The most similar 35 images of given query images D052 1 and D101_1. 


\section{Appendix-Table 1: Glossary of Notation}

The set of positive integers

A regular lattice

A pixel with coordinate $(i, j)$

$x_{i, j}$

The gray value of the image at position $(i, j)$

$\eta^{d}$

$d^{\text {th }}$ order neighborhood system

$\eta_{i j}^{d}$

The neighboring set of a pixel $i j$ in the $\mathrm{d}$.th order neighborhood system

$D(i j, k l) \quad$ The distance function between pixel $i j$ and $k l$

$\bar{p}, \bar{c}$

A position vector between two locations of a lattice, also called base clique type

$P$

Base clique type set

$<\bar{c}>$

An ordered tuple of base clique types

$B_{\bar{p}}(i j, k l) \quad$ Base Clique Test Predicate

$C_{L}^{\bar{p}}(i j) \quad$ A Clique chain with length $\mathrm{L}$ defined on a given seed pixel $i j$

$W_{S, L}^{\bar{p}<\bar{c}>}(a b) \quad$ A Clique Window with seed pixel $a b$. It consists of S clique chains $C_{L}^{\bar{p}}(i j)$

$W_{S, L}^{\bar{p}, \bar{c}}(a b) \quad$ A Regular Clique Window

$\bar{v}=\left(v_{x}, v_{y}\right) \quad$ A lag vector

$r(\bar{v})^{W_{S, L}^{\bar{p}, \bar{c}}(a b)} \quad$ Clique autocorrelation coefficient at lag vector $\bar{v}$ of a given clique window $W_{S, L}^{\bar{p}, \bar{c}}(a b)$

$N_{W_{S, L}^{\bar{c} . \bar{c}}(a b)} \quad$ The number of pixels in the clique window $W_{S, L}^{\bar{p}, \bar{c}}(a b)$

$\bar{x}_{i, j} \quad$ The mean value of the gray levels in the given clique window $W_{S, L}^{\bar{p}, \bar{c}}(a b)$

$\mu_{\mathrm{S}, \mathrm{L}}^{\overline{\mathrm{p}}, \overline{\mathrm{c}}}(\bar{v}) \quad$ Mean value of clique autocorrelation coefficients with lag vector $\bar{v}$ of all $W_{S, L}^{\bar{p}, \bar{c}}(a b)$

$\sigma_{\mathrm{S}, \mathrm{L}}^{\overline{\mathrm{p}}, \overline{\mathrm{c}}}(\bar{v}) \quad$ Standard deviation of clique autocorrelation coefficients with lag vector $\bar{v}$ of all $W_{S, L}^{\bar{p}, \bar{c}}(a b)$

$D_{T} \quad$ A SASI descriptor

$D_{T}^{\prime} \quad$ A normalized SASI descriptor

$S\left(T_{1}, T_{2}\right) \quad$ A mathematical similarity metric between the textures $\mathbf{T}_{\mathbf{1}}$ and $\mathbf{T}_{\mathbf{2}}$ 
